hep-ph/0009287

\title{
NEUTRINO MASSES AND MIXING ANGLES IN A REALISTIC STRING-INSPIRED MODEL
}

\author{
S. F. King and M.Oliveira \\ Department of Physics and Astronomy, University of Southampton \\ Southampton, SO17 1BJ, U.K
}

\begin{abstract}
We analyse a supersymmetric string-inspired model of all fermion masses and mixing angles based on the Pati-Salam $S U(4) \times S U(2)_{L} \times S U(2)_{R}$ gauge group supplemented by a $U(1)_{X}$ flavour symmetry. The model involves third family Yukawa unification and predicts the top mass and the ratio of the vacuum expectation values $\tan \beta$. The model also provides a successful description of the CKM matrix and predicts the masses of the down and strange quarks. However our main focus is on the neutrino masses and MNS mixing angles, and we show how the recent atmospheric neutrino mixing observed by Super-Kamiokande, and the MSW solution to the solar neutrino problem lead to important information about the flavour structure of the model near the string scale. We show how single right-handed neutrino dominance may be implemented by the use of "Clebsch zeros", leading to the LMA MSW solution, corresponding to bi-maximal mixing. The LOW MSW and SMA MSW solutions are also discussed.
\end{abstract}

October 25, 2018 


\section{INTRODUCTION}

The problem of understanding the quark and lepton masses and mixing angles represents one of the major unsolved questions of the standard model. Recently additional information on the fermion mass spectrum has come from the measurement of the atmospheric neutrino masses and mixing angles by Super-Kamiokande [四]. The most recent data disfavours mixing involving a sterile neutrino, and finds a good fit for $\nu_{\mu} \rightarrow \nu_{\tau}$ mixing with $\sin ^{2} 2 \theta_{23}>0.88$ and a mass square splitting $\Delta m_{23}^{2}$ in the $1.5-$ $5 \times 10^{-3} \mathrm{eV}^{2}$ range at $90 \% \mathrm{CL}$ [2]. Super-Kamiokande has also provided additional support for solar neutrino mixing. The most recent Super-Kamiokande data does not show a significant day-night asymmetry and shows an energy independent neutrino spectrum, thus it also disfavours the sterile neutrino mixing hypothesis, the just-so vacuum oscillation hypothesis, and the small mixing angle (SMA) MSW [3] solution [4]. The preferred solution at the present time seems to be the large mixing angle (LMA) MSW solution, although a similar solution with a low mass splitting (the LOW) solution is also possible. A typical point in the LMA MSW region is $\sin ^{2} 2 \theta_{12} \approx$ 0.75 , and $\Delta m_{12}^{2} \approx 2.5 \times 10^{-5} \mathrm{eV}^{2}$ 河.

If one accepts the recent data as evidence for neutrino masses and mixing angles, then the obvious question is how these can be accommodated in the standard model, or one of its supersymmetric extensions. The simplest possibility to account for the smallness of the neutrino masses is the see-saw mechanism [6] in which one introduces right-handed neutrinos which acquire very large Majorana masses at a super-heavy mass scale. When one integrates out the right-handed neutrinos the "normal sized" Dirac Yukawa couplings, which connect the left-handed to the right-handed neutrinos, are transformed into very small couplings which generate very light effective left-handed physical Majorana neutrino masses. Given the see-saw mechanism, it is natural to expect that the spectrum of the neutrino masses will be hierarchical, since 
the Dirac Yukawa couplings in the charged fermion sector are observed to be hierarchical, and if they are related to the Dirac neutrino Yukawa couplings then they should also be hierarchical, leading to hierarchical light Majorana masses. H

Having assumed the see-saw mechanism and a hierarchical neutrino mass spectrum, the next question is how such large (almost maximal) lepton mixing angles such as $\theta_{23}$ could emerge? There are several possibilities that have been suggested in the literature. One possibility is that it happens as a result of the off-diagonal 23 entries in the left-handed Majorana matrix being large, and the determinant of the 23 sub-matrix being accidentally small, leading to a neutrino mass hierarchy with large neutrino mixing angles [8]. Another possibility is that the neutrino mixing angles start out small at some high energy scale, then get magnified by renormalization group (RG) running down to low energies [9]. A third possibility is that the off-diagonal elements of the left-handed neutrino Majorana matrix are large, but the 23 sub-determinant of the matrix is small for a physical reason, as would be the case if a single right-handed neutrino were providing the dominant contribution to the 23 sub-matrix [10, 11, 12]. We shall refer to these three approaches as the accidental, the magnification and the single right-handed neutrino dominance (SRHND) mechanisms, respectively. As we shall see, in the model under consideration, only the SRHND mechanism provides a successful description of the atmospheric neutrino data, and the results in this paper will rely on this mechanism.

A promising approach to understanding the fermion mass spectrum is within the framework of supersymmetric (SUSY) unified theories. Within the framework of such theories the quark and lepton masses and mixing angles become related to each other, and it begins to be possible to understand the spectrum. The simplest grand unified theory (GUT) is $S U(5)$ but this theory in its minimal version does not contain any

\footnotetext{
${ }^{1}$ However this is not guaranteed due to the unknown structure of the heavy Majorana matrix, and for example an inverted neutrino mass hierarchy could result although this relies on some nonhierarchical couplings in the Dirac Yukawa matrix [7].
} 
right-handed neutrinos. Nevertheless three right-handed neutrinos may be added, and in this theory it is possible to have a large 23 element 7 on the Dirac neutrino Yukawa matrix without introducing a large 23 element into any of the charged fermion Yukawa matrices. The problem of maintaining a 23 neutrino mass hierarchy in these models may be solved for example by assuming SRHND [13]. Another possibility within the framework of $S U(5)$ is to maintain all the off-diagonal elements to be small, but require the 22 and 32 elements of the Dirac neutrino Yukawa matrix to be equal and the second right-handed neutrino to be dominant, in which case SRHND again leads to a large 23 neutrino mixing angle with hierarchical neutrino masses [14]. However the drawback of $S U(5)$ is that it does not predict any right-handed neutrinos, which must be added as an afterthought.

From the point of view of neutrino masses, the most natural GUTs are those like $S O(10)$ that naturally predict right-handed neutrinos. However within the framework of $S O(10)$ the quark masses and mixing angles are related to the lepton masses and mixing angles, and the existence of large neutrino mixing angles is not expected in the minimal versions of the theory in which the Higgs doublets are in one (or two) $\mathbf{1 0}^{\prime} s$ (ten dimensional representations of $S O(10)$ ) and each matter family is in a $\mathbf{1 6 .}$ Nevertheless various possibilities have been proposed in $S O(10)$ in order to account for the large neutrino mixing angles. Within the framework of minimal $S O(10)$ with third family Yukawa unification, it has been suggested that if two operators with different Clebsch coefficients contribute with similar strength then, with suitable choice of phases, in the case of the lepton Yukawa matrices one may have large numerical 23 elements, which add up to give a large lepton mixing angle, while for the quarks the 23 elements can be small due to approximate cancellation of the two contributing operators [15]. This is an example of the accidental mechanism mentioned above, where in addition one requires the quark mixing angles to be small by accident, although it

\footnotetext{
${ }^{2}$ We use Left-Right (LR) convention for Yukawa matrices in this paper.
} 
remains to be seen if the LMA MSW solution could be understood in this framework. Moving away from minimal $S O(10)$, one may invoke a non-minimal Higgs sector in which one Higgs doublet arises from a 10 and one from a 16, and in this framework it is possible to understand atmospheric neutrino mixing [16]. Alternatively, one may invoke a non-minimal matter sector in which parts of a quark and lepton family arise from a 16 and other parts from a 10, and in these models one may account for atmospheric and solar neutrinos via an inverted mass hierarchy mechanism [17].

In the present paper we shall discuss neutrino masses and mixing angles in a particular string-inspired minimal model based on the Pati-Salam $S U(4) \times S U(2)_{L} \times S U(2)_{R}$ (422) group [18]. As in $S O(10)$ the presence of the gauged $S U(2)_{R}$ predicts the existence of three right-handed neutrinos. However, unlike $S O(10)$, there is no Higgs doublet-triplet splitting problem since in the minimal model both Higgs doublets are contained in a $(1,2,2)$ representation. Moreover, since the left-handed quarks and leptons are in the $(4,2,1)$ and the right-handed quarks and leptons in the $(4,1,2)$ representations, the model also leads to third family Yukawa unification as in minimal $S O(10)$. Although the Pati-Salam gauge group is not unified at the field theory level, it readily emerges from string constructions either in the perturbative fermionic constructions [19], or in the more recent type I string constructions [20], unlike $S O(10)$ which typically requires large Higgs representations which do not arise from the simplest string constructions. The question of fermion masses and mixing angles in the string-inspired Pati-Salam model has already been discussed for the case of charged fermions [21, 22], and later for the case of neutrinos [23]. For the neutrino study [23] it was assumed that the heavy Majorana neutrino mass matrix was proportional to the unit matrix, and only small neutrino mixing angles were considered. Later on a $U(1)_{X}$ family symmetry was added to the model, in order to understand the horizontal hierarchies, although in this case the neutrino spectrum was not analysed at all [24. 
The purpose of the present paper is to discuss neutrino masses and mixing angles in the string-inspired Pati-Salam model supplemented by a $U(1)_{X}$ flavour symmetry. The model involves third family Yukawa unification and predicts the top mass and the ratio of the vacuum expectation values $\tan \beta$, as we recently discussed in Ref. [25]. It is already known that the model can provide a successful description of the CKM matrix and predicts the down and strange quark masses, although our present analysis differs from that presented previously [24] partly due to the recent refinements in third family Yukawa unification [25], but mainly as a result of the recent Super-Kamiokande data which has important implications for the flavour structure of the model. In fact our main focus here is on the neutrino masses and mixing angles which were not previously discussed at all in this framework. We assume a minimal version of the model, and avoid the use of the accidental cancellation mechanism, which in any case has difficulties in accounting for bi-maximal neutrino mixing. We also show that the mixing angle magnification mechanism can only provide limited increases in the mixing angles, due to the fact that the unified third family Yukawa coupling is only approximately equal to 0.7 [25] and is therefore too small to have a dramatic effect. Instead, we rely on the SRHND mechanism, and we show how this mechanism may be implemented in the 422 model by appropriate use of operators with "Clebsch zeros" resulting in a natural explanation for atmospheric neutrinos via a hierarchical mass spectrum. We specifically focus on the LMA MSW solution in the text, with the LOW and SMA MSW solutions relegated to Appendices.

The layout of the remainder of the paper is as follows. In section II we briefly review the see-saw mechanism in the Minimal Supersymmetric Standard Model (MSSM) [26] with right-handed neutrinos. In section III we review some useful analytic results for SRHND, for the case of an approximately diagonal right-handed Majorana mass matrix. In section IV we introduce the string-inspired Pati-Salam model, and in section $\mathrm{V}$ we introduce an Abelian anomalous gauge $U(1)_{X}$ family symmetry into 
the model, and show how horizontal Yukawa hierarchies may be generated. In section VI we describe our operator approach to fermion masses, including the heavy Majorana neutrino masses. Section VII contains the main results of the paper. In this section we show how a particular choice of $U(1)_{X}$ family charges, and operators with certain Clebsch coefficients can lead to a successful description of quark and lepton masses and mixing angles, and in particular describe atmospheric and solar neutrinos via SRHND. Although the neutrino masses and mixing angles correspond to the usual LMA MSW solution, in Appendix C we show how a modification of the heavy Majorana mass matrix can lead to a large mixing angle MSW solution with a LOW mass splitting. In Appendix D we present a different choice of $U(1)_{X}$ charges and operators which can lead to the SMA MSW solution.

\section{THE MSSM WITH RIGHT-HANDED NEUTRINOS}

The superpotential of the MSSM with right-handed neutrinos is given by :

$$
\begin{aligned}
\mathcal{W} & =\mathcal{W}_{M S S M}+\mathcal{W}_{\nu^{c}} \\
\mathcal{W}_{M S S M} & =q_{A}\left(\lambda_{u}\right)_{A B} u_{B}^{c} h_{u}-q_{A}\left(\lambda_{d}\right)_{A B} d_{B}^{c} h_{d}-l_{A}\left(\lambda_{e}\right)_{A B} e_{B}^{c} h_{d}+\mu h_{u} h_{d} \\
\mathcal{W}_{\nu^{c}} & =l_{A}\left(\lambda_{\nu}\right)_{A B} \nu_{B}^{c} h_{u}+\frac{1}{2} \nu_{A}^{c}\left(M_{R R}\right)_{A B} \nu_{B}^{c}
\end{aligned}
$$

where $A, B=1, . ., 3$ are family indices, $u^{c}, d^{c}, e^{c}$ and $\nu^{c}$ are the right-handed $S U(2)_{L}$ singlet superfields, $q=(u, d)$ and $l=(\nu, e)$ are the $S U(2)_{L}$ quark and lepton doublets, and $h_{u}\left(h_{d}\right)$ is the up (down) Higgs boson doublet. The Dirac neutrino coupling and the heavy Majorana mass for the right-handed neutrinos are denoted by $\lambda_{\nu}$ and $M_{R R}$

respectively. When the neutral components of the two MSSM Higgs bosons $h_{u, d}^{0}$ acquire their vacuum expectation values $(\mathrm{VEV}) \mathrm{s} v_{2,1}\left(\tan \beta=v_{2} / v_{1} \sim 40-50\right)$ the superpotential in Eq. (11) generates the following sum of mass terms :

$$
\mathcal{L}_{U, D, E}=-U\left(\lambda_{u} v_{2}\right) U^{c}-D\left(\lambda_{d} v_{1}\right) D^{c}-E\left(\lambda_{e} v_{1}\right) E^{c}+\text { h.c. }
$$




$$
\mathcal{L}_{N}=-N\left(\lambda_{\nu} v_{2}\right) N^{c}-\frac{1}{2} N^{c} M_{R R} N^{c}+\text { h.c. }
$$

where the upper case letters now denote the fermionic components of the superfields in $\mathcal{W}$, for example $u$ contains $(U, \tilde{u}) \equiv\left(U_{L}, \tilde{u}_{L}\right)$ and $u^{c}$ contains $\left(U^{c}, \tilde{u}^{c}\right) \equiv\left(U_{R}^{*}, \tilde{u}_{R}^{*}\right)$. The Yukawa matrices in Eq. (田) can be diagonalized by bi-unitary transformations $S$ and $T$ defined by :

$$
T^{u *} \lambda_{u} S^{u T}=\lambda_{u}^{\prime} \quad T^{d *} \lambda_{d} S^{d T}=\lambda_{d}^{\prime} \quad T^{e *} \lambda_{e} S^{e T}=\lambda_{e}^{\prime}
$$

Thus the physical (primed) states $U_{R, L}^{\prime}$ are related to the gauge eigenstates $U_{R, L}$ by $U_{R}^{\prime}=S^{u} U_{R}$ and $U_{L}^{\prime}=T^{u} U_{L}$, etc.. In this model, the left-handed neutrino masses are generated via see-saw mechanism [6] by the terms in Eq. (5) which can be re-arranged into a two-by-two block matrix in the following way :

$$
\mathcal{L}_{N}=-\frac{1}{2}\left(N N^{c}\right)\left(\begin{array}{cc}
0 & m_{L R} \\
m_{L R}^{T} & M_{R R}
\end{array}\right)\left(\begin{array}{c}
N \\
N^{c}
\end{array}\right)+\text { h.c. }
$$

where $m_{L R}=\lambda_{\nu} v_{2}$. Thus, after the heavy $N^{c}$ fields are integrated out, the light left-handed neutrinos $N$ effectively acquire a small mass given by :

$$
m_{L L}=m_{L R} M_{R R}^{-1} m_{L R}^{T}
$$

Finally, the diagonalization of $m_{L L}$ :

$$
T^{N *} m_{L L} T_{L}^{N \dagger}=\operatorname{diag}\left(m_{\nu_{1}}, m_{\nu_{2}}, m_{\nu_{3}}\right)
$$

allows the determination of the masses of the physical neutrinos $m_{\nu_{A}}$ and enables the physical neutrino states $N^{\prime}=\left(\nu_{1}, \nu_{2}, \nu_{3}\right)$ to be related to the neutrino gauge fields $N=\left(\nu_{e}, \nu_{\mu}, \nu_{\tau}\right)$ by $N^{\prime}=T^{N} N$.

Taking into account the above conventions, we now proceed to give expressions for the Cabbibo-Kobayashi-Maskawa (CKM) matrix [27] $\left(V^{C K M}\right)$ and the corresponding lepton analogue, the Maki-Nakawaga-Sakata (MNS) matrix [28] $\left(V^{M N S}\right)$. Their 
definitions derive from the charged current interactions : [ ]

$$
\begin{gathered}
-\frac{g}{\sqrt{2}} W_{\mu}^{+} \bar{\Psi}_{U} \gamma^{\mu} P_{L} \Psi_{D} \rightarrow-\frac{g}{\sqrt{2}} W_{\mu}^{+} \Psi_{U^{\prime}} \gamma^{\mu} P_{L} V^{C K M} \Psi_{D^{\prime}} \\
-\frac{g}{\sqrt{2}} W_{\mu}^{-} \bar{\Psi}_{E} \gamma^{\mu} P_{L} \Psi_{N} \rightarrow-\frac{g}{\sqrt{2}} W_{\mu}^{-} \bar{\Psi}_{E^{\prime}} \gamma^{\mu} P_{L} V^{M N S} \Psi_{N^{\prime}}
\end{gathered}
$$

that imply :

$$
V^{C K M}=T^{u} T^{d \dagger} \quad V^{M N S}=T^{e} T^{N \dagger}
$$

In what follows we will assume that the matrices in Eq. (12) are real. 1 Thus, we will write $V^{M N S}$ in terms of three rotation matrices :

$$
V^{M N S}=R_{23} R_{13} R_{12}
$$

given by :

$$
R_{23}=\left(\begin{array}{ccc}
1 & 0 & 0 \\
0 & c_{23} & s_{23} \\
0 & -s_{23} & c_{23}
\end{array}\right) \quad R_{13}=\left(\begin{array}{ccc}
c_{13} & 0 & s_{13} \\
0 & 1 & 0 \\
-s_{13} & 0 & c_{13}
\end{array}\right) \quad R_{12}=\left(\begin{array}{ccc}
c_{12} & s_{12} & 0 \\
-s_{12} & c_{12} & 0 \\
0 & 0 & 1
\end{array}\right)
$$

where $s_{A B}=\sin \theta_{A B}, c_{A B}=\cos \theta_{A B}$ refer to the lepton mixing angles between the $A$ and $B$ generation. Using Eq. (14) into Eq. (13) gives :

$$
V^{M N S}=\left(\begin{array}{ccc}
c_{12} c_{13} & s_{12} c_{13} & s_{13} \\
-s_{12} c_{23}-c_{12} s_{23} s_{13} & c_{12} c_{23}-s_{12} s_{23} s_{13} & s_{23} c_{13} \\
s_{12} s_{23}-c_{12} c_{23} s_{13} & -c_{12} s_{23}-s_{12} c_{23} s_{13} & c_{23} c_{13}
\end{array}\right)
$$

It is also practical to have expressions for the $\theta_{A B}$ angles in terms of the $V^{M N S}$ entries. Inverting Eq. (15) we find that : f

$$
\sin \theta_{13}=V_{e 3} \quad \sin \theta_{23}=\frac{V_{\mu 3}}{\sqrt{1-V_{e 3}^{2}}} \quad \sin \theta_{12}=\frac{V_{e 2}}{\sqrt{1-V_{e 3}^{2}}}
$$

Finally we note that while the above expressions were derived in the context of three neutrino species, the analysis of the experimental results assumed only two, thus a direct comparison of mixing angles is not exactly valid.

\footnotetext{
${ }^{3}$ The four component fermion fields $\Psi$ are given by $\Psi_{F}=\left(F,-i \sigma^{2} F^{c *}\right)$ for $F=U, D, E$ and $\Psi_{N}=\left(N,-i \sigma^{2} N^{*}\right)$ for the neutrinos.

${ }^{4} \mathrm{We}$ shall not address the question of $\mathrm{CP}$ violation in this paper.

${ }^{5} V_{e 2}=V_{12}^{M N S}, V_{e 3}=V_{13}^{M N S}$ and $V_{\mu 3}=V_{23}^{M N S}$.
} 


\section{SINGLE RIGHT-HANDED NEUTRINO DOMINANCE}

Third family single right-handed neutrino dominance (SRHND) [10, 11, 12] is a mechanism that can explain the large atmospheric $\left(\theta_{23}\right)$ and the solar LMA MSW $\left(\theta_{12}\right)$ neutrino mixing angles and a small $\theta_{13}$. SRHND relies on the possibility that the neutrino mass matrix $\left(m_{L L}\right)$ is dominated by the contributions coming solely from a single right-handed neutrino (for example $\nu_{\tau}^{c}$.) In this scheme a maximal $\theta_{23}$ angle arises when the tau right-handed neutrino $\nu_{\tau}^{c}$ couples to the left-handed muon $\nu_{\mu}$ and tau neutrino $\nu_{\tau}$ with equal strength. Similarly, if $\nu_{\mu}^{c}$ couples to $\nu_{e}$ and to $\nu_{\mu}$ with comparable strength then $\theta_{12}$ is large. The role of the (sub-dominant) muon neutrino is also important since it provides small perturbations to the $m_{L L}$ matrix (which otherwise has one heavy and two massless eigenstates), thus leading to a neutrino mass splitting $\Delta m_{12}^{2}=\left|m_{\nu_{2}}^{2}-m_{\nu_{1}}^{2}\right|$ compatible with experiment. In this section we summarize the theory behind SRHND and review the analytic results presented in Ref. [12] for the case of the diagonal dominated right-handed neutrino mass matrix $M_{R R}$.

The see-saw formula for the left-handed neutrino matrix in Eq. (8) depends explicitly on $M_{R R}$. Although $M_{R R}$ might have a non-trivial structure we find instructive to start our analysis by considering the very simple case of $M_{R R}$ given by :

$$
M_{R R}^{-1} \sim \operatorname{diag}\left(M_{\nu_{1}}^{-1}, M_{\nu_{2}}^{-1}, M_{\nu_{3}}^{-1}\right) \sim \operatorname{diag}\left(0,0, M_{\nu_{3}}^{-1}\right)
$$

which effectively corresponds to taking $M_{\nu_{1}}, M_{\nu_{2}} \gg M_{\nu_{3}}$. Replacing Eq. (17) into Eq. (8) we find that: 9

$$
m_{L L}=v_{2}^{2} \lambda_{\nu} M_{R R}^{-1} \lambda_{\nu}^{T} \sim \lambda M_{R R}^{-1} \lambda^{T} \sim M_{\nu_{3}}^{-1}\left(\begin{array}{ccc}
\lambda_{13}^{2} & \lambda_{13} \lambda_{23} & \lambda_{13} \lambda_{33} \\
\lambda_{13} \lambda_{23} & \lambda_{22}^{2} & \lambda_{23} \lambda_{33} \\
\lambda_{13} \lambda_{33} & \lambda_{23} \lambda_{33} & \lambda_{33}^{2}
\end{array}\right)
$$

The $m_{L L}$ matrix above is easily diagonalized by the matrices $R_{23}, R_{13}, R_{12}$ 【 in

\footnotetext{
${ }^{6}$ In this section we will use the following simplified notation $m_{L R}=\lambda_{\nu} v_{2} \equiv \lambda v_{2} \sim \lambda$.

${ }^{7}$ Note that $R_{12}$ for $m_{L L}$ in Eq. (18) is undetermined.
} 
Eq. (14) with rotation angles given by :

$$
\begin{array}{lll}
s_{23}=\frac{\lambda_{23}}{A} & c_{23}=\frac{\lambda_{33}}{A} & A^{2}=\lambda_{33}^{2}+\lambda_{23}^{2} \\
s_{13}=\frac{\lambda_{13}}{B} & c_{13}=\frac{A}{B} & B^{2}=\lambda_{33}^{2}+\lambda_{23}^{2}+\lambda_{13}^{2}
\end{array}
$$

that successively act on $m_{L L}$ as follows :

$$
m_{L L}^{\prime \prime \prime}=R_{12}^{\dagger} R_{13}^{\dagger} R_{23}^{\dagger} m_{L L} R_{23} R_{13} R_{12}=\operatorname{diag}\left(m_{\nu_{1}}, m_{\nu_{2}}, m_{\nu_{3}}\right)
$$

It is also convenient to define the following primed matrices :

$$
m_{L L}^{\prime}=R_{23}^{\dagger} m_{L L} R_{23} \quad m_{L L}^{\prime \prime}=R_{13}^{\dagger} m_{L L}^{\prime} R_{13} \quad m_{L L}^{\prime \prime \prime}=R_{12}^{\dagger} m_{L L}^{\prime \prime} R_{12}
$$

which, for $m_{L L}$ as in Eq. (18), are explicitly given by :

$$
m_{L L}^{\prime} \sim M_{\nu_{3}}^{-1}\left(\begin{array}{ccc}
\lambda_{13}^{2} & 0 & \lambda_{13} A \\
0 & 1 & 0 \\
\lambda_{13} A & 0 & A^{2}
\end{array}\right) \quad m_{L L}^{\prime \prime} \equiv m_{L L}^{\prime \prime \prime} \sim M_{\nu_{3}}^{-1}\left(\begin{array}{ccc}
0 & 0 & 0 \\
0 & 0 & 0 \\
0 & 0 & B^{2}
\end{array}\right)
$$

We can see from Eq. (19) that if $\lambda_{23}=\lambda_{33}$ then a maximal $\theta_{23}=45^{0}$ angle results. Moreover, if $\lambda_{13} \ll \lambda_{23}, \lambda_{33}$ then $\theta_{13}$ is small. Although SRHND, in the limiting case of Eq. (17), is successful in predicting a maximal atmospheric neutrino angle, it fails to account for a viable neutrino spectrum. Indeed, from Eq. (22), we see that the two lightest neutrinos are massless $m_{\nu_{1}}=m_{\nu_{2}}=0$. Moreover the solar neutrino angle $\theta_{12}$ is undetermined. These two problems can be solved by allowing the right-handed muon neutrino $\nu_{\mu}^{c}$ to play a sub-dominant/perturbative role in the structure of $m_{L L}$ in Eq. (18).

We now turn to the more realist model in which $M_{R R}$ can be approximated by : 8

$$
M_{R R}^{-1} \sim \operatorname{diag}\left(M_{\nu_{1}}^{-1}, M_{\nu_{2}}^{-1}, M_{\nu_{3}}^{-1}\right) \sim \operatorname{diag}\left(0, M_{\nu_{2}}^{-1}, M_{\nu_{3}}^{-1}\right)
$$

Using Eq. (23) into Eq. (8) we find that :

\footnotetext{
${ }^{8}$ Note that although Eq. (23) still looks very simple it can, in many cases, provide a good qualitative description of the physics involving the heaviest neutrinos. Indeed, if $M_{R R}$ is diagonal dominated and if $m_{R L}$ is highly hierarchical then the limiting case of Eq. (23) applies.
} 


$$
m_{L L} \sim M_{\nu_{3}}^{-1}\left(\begin{array}{ccc}
\lambda_{13}^{2} & \lambda_{13} \lambda_{23} & \lambda_{13} \lambda_{33} \\
\lambda_{13} \lambda_{23} & \lambda_{23}^{2} & \lambda_{23} \lambda_{33} \\
\lambda_{13} \lambda_{33} & \lambda_{23} \lambda_{33} & \lambda_{33}^{2}
\end{array}\right)+M_{\nu_{2}}^{-1}\left(\begin{array}{ccc}
\lambda_{12}^{2} & \lambda_{12} \lambda_{22} & \lambda_{12} \lambda_{32} \\
\lambda_{12} \lambda_{22} & \lambda_{22}^{2} & \lambda_{22} \lambda_{32} \\
\lambda_{12} \lambda_{32} & \lambda_{22} \lambda_{32} & \lambda_{32}^{2}
\end{array}\right)
$$

Given that we assumed SRHND by the $\nu_{\tau}^{c}$ neutrino, it follows that the contributions to the 23 block of $m_{L L}$ in Eq. (24) arising from the terms proportional to $M_{\nu_{3}}^{-1}$ dominate over the ones proportional to $M_{\nu_{2}}^{-1}$. I Clearly, the rotations $R_{12}, R_{13}$ parameterised by the angles in Eq. (19) diagonalize $m_{L L}$ in Eq. (24) up to terms of order $\mathcal{O}\left(M_{\nu_{2}}^{-1}\right)$. Thus the new primed matrices $m_{L L}^{\prime}$ and $m_{L L}^{\prime \prime}$ are given by :

$$
\begin{aligned}
& m_{L L}^{\prime} \sim M_{\nu_{3}}^{-1}\left(\begin{array}{ccc}
\lambda_{13}^{2} & 0 & \lambda_{13} A \\
0 & 0 & 0 \\
\lambda_{13} A & 0 & A^{2}
\end{array}\right)+M_{\nu_{2}}^{-1}\left(\begin{array}{ccc}
\lambda_{12}^{2} & \lambda_{12} \frac{C^{2}}{A} & \lambda_{12} \frac{D^{2}}{A} \\
\lambda_{12} \frac{C^{2}}{A} & \frac{C^{4}}{A^{2}} & \frac{C^{2} D^{2}}{A^{2}} \\
\lambda_{12} \frac{D^{2}}{A} & \frac{C^{2} D^{2}}{A^{2}} & \frac{D^{4}}{A^{2}}
\end{array}\right) \\
& m_{L L}^{\prime \prime} \sim M_{\nu_{3}}^{-1}\left(\begin{array}{ccc}
0 & 0 & 0 \\
0 & 0 & 0 \\
0 & 0 & B^{2}
\end{array}\right)+M_{\nu_{2}}^{-1}\left(\begin{array}{ccc}
\frac{E^{6}}{A^{2} B^{2}} & \frac{C^{2} E^{3}}{A^{2} E^{2}} & \frac{F^{2} E^{3}}{A B^{2}} \\
\frac{C^{2}}{A^{2} B} & \frac{C^{4}}{A^{2}} & \frac{C^{2} F^{2}}{A B} \\
\frac{F^{2} E^{3}}{A B^{2}} & \frac{C^{2} F^{2}}{A B} & \frac{F^{4}}{B^{2}}
\end{array}\right)
\end{aligned}
$$

where

$$
\begin{aligned}
& C^{2}=\lambda_{22} \lambda_{33}-\lambda_{32} \lambda_{23} \\
& D^{2}=\lambda_{33} \lambda_{32}+\lambda_{22} \lambda_{23} \\
& E^{3}=\lambda_{12}\left(\lambda_{33}^{2}+\lambda_{23}^{2}\right)-\lambda_{13}\left(\lambda_{33} \lambda_{32}+\lambda_{22} \lambda_{23}\right) \\
& F^{2}=\lambda_{33} \lambda_{32}+\lambda_{22} \lambda_{23}+\lambda_{12} \lambda_{13}
\end{aligned}
$$

The diagonalization of the 12 block of $m_{L L}^{\prime \prime}$ in Eq. (26) is achieved by a $R_{12}$ matrix parameterised by the following $\theta_{12}$ rotation angle :

$$
s_{12}=\frac{E^{3}}{\sqrt{E^{6}+B^{2} C^{4}}} \quad c_{12}=\frac{B C^{2}}{\sqrt{E^{6}+B^{2} C^{4}}}
$$

Thus we find :

$$
m_{L L}^{\prime \prime \prime} \sim M_{\nu_{3}}^{-1}\left(\begin{array}{ccc}
0 & 0 & 0 \\
0 & 0 & 0 \\
0 & 0 & B^{2}
\end{array}\right)+M_{\nu_{2}}^{-1}\left(\begin{array}{ccc}
0 & 0 & 0 \\
0 & \frac{E^{6}+B^{2} C^{4}}{A A^{2} B^{2}} & \frac{F^{2} \sqrt{E^{6}+B^{2} C^{4}}}{A B^{2}} \\
0 & \frac{F^{2} \sqrt{E^{6}+B^{2} C^{4}}}{A B^{2}} & \frac{F^{4}}{B^{2}}
\end{array}\right)
$$

\footnotetext{
${ }^{9}$ Note that this does not necessarily imply that $M_{\nu_{3}}^{-1}$ is larger than $M_{\nu_{2}}^{-1}$ since the Yukawa couplings must also be taken into account.
} 
It is interesting to note that the $R_{12}$ rotation has not only diagonalized the 12 block of $m_{L L}^{\prime \prime}$ but also put zeros in the 13,31 entries of $m_{L L}^{\prime \prime \prime}$. The reason is because $m_{L L}^{\prime \prime}$ displays a special structure. Indeed, their elements obey :

$$
t_{12}=\frac{s_{12}}{c_{12}}=\frac{\left(m_{L L}^{\prime \prime}\right)_{12}}{\left(m_{L L}^{\prime \prime}\right)_{22}}=\frac{\left(m_{L L}^{\prime \prime}\right)_{11}}{\left(m_{L L}^{\prime \prime}\right)_{12}}=\frac{\left(m_{L L}^{\prime \prime}\right)_{13}}{\left(m_{L L}^{\prime \prime}\right)_{23}}=\frac{E^{3}}{B C^{2}}
$$

Explicitly $t_{12}=\tan \theta_{12}$ is given by :

$$
t_{12}=\frac{\lambda_{12}\left(\lambda_{33}^{2}+\lambda_{23}^{2}\right)-\lambda_{13}\left(\lambda_{33} \lambda_{32}+\lambda_{22} \lambda_{23}\right)}{\left(\lambda_{22} \lambda_{33}-\lambda_{32} \lambda_{23}\right) \sqrt{\lambda_{33}^{2}+\lambda_{23}^{2}+\lambda_{13}^{2}}} \sim \frac{\lambda_{12}}{\lambda_{22}}
$$

From Eq. (34) we see that, although $t_{12}$ generally depends on the second and third family neutrino Yukawa couplings, if $\lambda_{33}$ is much bigger than the other Yukawa couplings then $t_{12} \sim \lambda_{12} / \lambda_{22}$. This means that the $\theta_{12}$ angle is set not by the dominant neutrino couplings, but by the sub-dominant $\nu_{\mu}^{c}$ neutrino couplings to the $\nu_{e}$ and $\nu_{\mu}$ neutrinos. Thus while a large atmospheric neutrino mixing angle $\theta_{23}$ can be achieved by requiring $\lambda_{23} \sim \lambda_{33}$, a large MSW solar neutrino angle $\theta_{12}$ results from $\lambda_{12} \sim \lambda_{22}$. Moreover, Eq. (19) and Eq. (34) show that bi-maximal $\theta_{23}, \theta_{12}$ mixing can be achieved with a small $\theta_{13}$ angle as long as $\lambda_{13} \ll \lambda_{23}, \lambda_{33}$. The neutrino mass spectrum can be read from $m_{L L}^{\prime \prime \prime}$ in Eq. (32). We find a massless neutrino state $m_{\nu_{1}}=0$, plus a light state with mass $m_{\nu_{2}} \sim \lambda_{22}^{2} / M_{\nu_{2}}$ and a heavy neutrino with mass $m_{\nu_{3}} \sim \lambda_{33}^{2} / M_{\nu_{3}}$.

\section{THE PATI-SALAM MODEL}

Here we briefly summarize the parts of the Pati-Salam model [18 that are relevant for our analysis. For a more complete discussion see Ref. [19]. The SM fermions, together with the right-handed neutrinos, are conveniently accommodated in the following $F=(4,2,1)$ and $F^{c}=(\overline{4}, 1, \overline{2})$ representations :

$$
F_{A}=\left(\begin{array}{llll}
u & u & u & \nu \\
d & d & d & e
\end{array}\right)_{A} \quad F_{B}^{c}=\left(\begin{array}{llll}
d^{c} & d^{c} & d^{c} & e^{c} \\
u^{c} & u^{c} & u^{c} & \nu^{c}
\end{array}\right)_{B}
$$

The MSSM Higgs bosons fields are contained in $h=(1, \overline{2}, 2)$ :

$$
h=\left(\begin{array}{cc}
h_{d}^{-} & h_{u}^{0} \\
h_{d}^{0} & h_{u}^{+}
\end{array}\right)
$$


whereas the heavy Higgs bosons $\bar{H}=(\overline{4}, 1, \overline{2})$ and $H=(4,1,2)$ are denoted by :

$$
\bar{H}=\left(\begin{array}{cccc}
\bar{H}_{d} & \bar{H}_{d} & \bar{H}_{d} & \bar{H}_{e} \\
\bar{H}_{u} & \bar{H}_{u} & \bar{H}_{u} & \bar{H}_{\nu}
\end{array}\right) \quad H=\left(\begin{array}{cccc}
H_{d} & H_{d} & H_{d} & H_{e} \\
H_{u} & H_{u} & H_{u} & H_{\nu}
\end{array}\right) .
$$

In addition to the Higgs fields in Eqs. (36),(37) the model also involves an $S U(4)$ sextet field $D=(6,1,1)=\left(D_{3}, D_{3}^{c}\right)$.

The superpotential of the minimal 422 model is :

$$
\begin{aligned}
\mathcal{W}= & F \lambda F^{c} h+\lambda_{h} S h h+ \\
& \lambda_{S} S\left(\bar{H} H-M_{H}^{2}\right)+\lambda_{H} H H D+\lambda_{\bar{H}} \bar{H} \bar{H} D+F^{c} \lambda^{\prime} F^{c} \frac{H H}{M_{V}}
\end{aligned}
$$

where $S$ denotes a gauge singlet superfield, the $\lambda$ 's are real dimensionless parameters and $M_{H} \sim M_{X} \sim 10^{16} \mathrm{GeV}$. Additionally, $M_{V}>M_{X}$ denotes the mass of extra exotic matter that has been integrated out from the model at high energy. As a result of the superpotential terms involving the singlet $S$, the Higgs fields develop VEVs $\langle H\rangle=\left\langle H_{\nu}\right\rangle \sim M_{X}$ and $\langle\bar{H}\rangle=\left\langle\bar{H}_{\nu}\right\rangle \sim M_{X}$ which lead to the symmetry breaking :

$$
S U(4) \otimes S U(2)_{L} \otimes S U(2)_{R} \rightarrow S U(3)_{c} \otimes S U(2)_{L} \otimes U(1)_{Y}
$$

The singlet $S$ itself also naturally develops a small VEV of the order of the SUSY breaking scale [29] so that the $\lambda_{h} S$ term in Eq. (38) gives an effective $\mu$ parameter of the correct order of magnitude. Under Eq. (39) the Higgs field $h$ in Eq. (36) splits into the familiar MSSM doublets $h_{u}$ and $h_{d}$ whose neutral components subsequently develop weak scale VEVs $\left\langle h_{u}^{0}\right\rangle=v_{2}$ and $\left\langle h_{d}^{0}\right\rangle=v_{1}$ with $\tan \beta=v_{2} / v_{1}$. The neutrino fields $\nu^{c}$ acquires a large mass $M_{R R} \sim \lambda^{\prime}\langle H H\rangle / M_{V}$ through the non-renormalizable term in $\mathcal{W}$ which, together with the Dirac $\nu^{c}-\nu$ interaction (proportional to $\lambda\left\langle h_{u}^{0}\right\rangle$ ), gives rise to a $2 \times 2$ matrix that generates, via see-saw mechanism [6], a suppressed mass for the left-handed neutrino states. The $D$ field does not develop a VEV but the terms $H H D$ and $\bar{H} \bar{H} D$ combine the colour triplet parts of $H, \bar{H}$ and $D$ into acceptable GUT scale mass terms [19. 


\section{ABELIAN FLAVOUR SYMMETRY}

The pattern of fermion masses and mixing angles is one of the fundamental problems is particle physics that has not yet been understood. The importance of this unsolved puzzle is demonstrated by the numerous works published in the literature over the past years (see Refs. [30]-36] for a "short" list.) In the standard model (SM) the quark/lepton masses and the CKM matrix are input parameters fixed by laboratory experiments. Surprisingly, however, their values, though unconstrained and a priori arbitrary, do display a certain degree of organization. The fermion masses are highly hierarchical and the CKM matrix can be described in terms of the small Wolfenstein expansion parameter $\lambda \sim\left|V_{12}\right| \sim 0.22$ [37]. These results suggest that a broken flavour symmetry might be playing an important role in the setting of the structure of the Yukawa matrices.

In this work we will assume that the "vertical" gauge group is supplemented by an additional $U(1)_{X}$ "horizontal" flavour symmetry that constraints the nature of the couplings of quarks and leptons to SM singlet fields $\theta$ and $\bar{\theta}$. The family symmetry, however, is broken at some high energy scale $M_{\theta}>M_{X}$ by the VEVs of the $\theta$, $\bar{\theta}$ fields which under the $U(1)_{X}$ group have charges $X_{\theta}=-1$ and $X_{\bar{\theta}}=+1$. As a consequence of the $U(1)_{X}$ symmetry breaking, the low energy effective theory includes Dirac interactions between the $F$ and $F^{c}$ fields of the following form :

$$
\begin{aligned}
& F_{A} F_{B}^{c} h\left(\frac{\theta}{M_{V}}\right)^{p_{A B}} \rightarrow F_{A} F_{B}^{c} h\left(\frac{\langle\theta\rangle}{M_{V}}\right)^{p_{A B}} \sim F_{A} F_{B}^{c} h \epsilon^{p_{A B}} \\
& F_{A} F_{B}^{c} h\left(\frac{\bar{\theta}}{M_{V}}\right)^{p_{A B}} \rightarrow F_{A} F_{B}^{c} h\left(\frac{\langle\bar{\theta}\rangle}{M_{V}}\right)^{p_{A B}} \sim F_{A} F_{B}^{c} h \epsilon^{p_{A B}}
\end{aligned}
$$

where $p_{A B}$ is the modulos of the sum of the $U(1)_{X}$ charges of the $F_{A}, F_{B}^{c}$ and $h$ fields, i.e. $p_{A B}=\left|X_{A B}\right|=\left|X_{F_{A}}+X_{F_{B}^{c}}+X_{h}\right|$. Thus Eq. (40) holds if $X_{A B}>0$ whereas Eq. (41) holds if $X_{A B}<0$. The non-renormalizable terms in Eqs. (40), (41) might originate from interactions between the $F$ and $\theta$ fields with additional exotic 
vector matter with mass $M_{V}>M_{X}$ that lead to "spaghetti" diagrams as discussed in Ref. [38]. In summary, the equations above show that, in the context of a $U(1)_{X}$ symmetry, the observed hierarchy in the fermion masses and mixing angles might be the result of the flavour charges carried by the fields of the 422 model which act to suppress the Yukawa couplings by some $\epsilon$-power.

The introduction of the $U(1)_{X}$ symmetry provides a way to relate the various flavour parameters of the model thus making it more predictive. However, one should be careful. Generally the $U(1)_{X}$ group is potentially dangerous since it can introduce, through triangle diagrams, mixed anomalies with the SM gauge group. In the last part of this section we review the constraints imposed on $X$ charges of the fields of our model enforced by the requirement of anomaly cancellation [32].

The mixed anomalies that we shall consider are : "m

$$
\begin{aligned}
S U(3)^{2} U(1)_{X} \quad: \quad A_{3}=\sum_{A=1}^{3}\left(2 X_{q_{A}}+X_{u_{A}^{c}}+X_{d_{A}^{c}}\right) \\
S U(2)^{2} U(1)_{X} \quad: \quad A_{2}=\sum_{A=1}^{3}\left(3 X_{q_{A}}+X_{l_{A}}\right)+X_{h_{u}}+X_{h_{d}} \\
U(1)_{Y}^{2} U(1)_{X} \quad: \quad A_{1}=\sum_{A=1}^{3}\left(\frac{1}{3} X_{q_{A}}+\frac{8}{3} X_{u_{A}^{c}}+\frac{2}{3} X_{d_{A}^{c}}+X_{l_{A}}+2 X_{e_{A}^{c}}\right)+X_{h_{u}}+X_{h_{d}} \\
U(1)_{Y} U(1)_{X}^{2} \quad: \quad A_{1}^{\prime}=\sum_{A=1}^{3}\left(X_{q_{A}}^{2}-2 X_{u_{A}^{c}}^{2}+X_{d_{A}^{c}}^{2}-X_{l_{A}}^{2}+X_{e_{A}^{c}}^{2}\right)+X_{h_{u}}^{2}+X_{h_{d}}^{2}
\end{aligned}
$$

For example, $A_{3}$ corresponds to the anomalous term generated by the Feynman diagram that has two $S U(3)$ gluons and one $U(1)_{X}$ gauge boson attached to the triangle vertices. We note that the first three anomalies $A_{3}, A_{2}$ and $A_{1}$ are linear in the trace of the charges, i.e. $X_{f}=\sum_{A=1}^{3} X_{f_{A}}$, where $f$ is any of the $q, u^{c}, d^{c}, l, e^{c}$ fields, thus they constraint only the family independent (FI) part of the $U(1)_{X}$ charges. On the other hand, $A_{1}^{\prime}$ is quadratic in the $X$ charges, thus it generally constraints the $\mathrm{FI}$ and

\footnotetext{
${ }^{10}$ The cancellation of anomalies requires the vanishing of the trace $\operatorname{Tr}\left(T^{a}\left\{T^{b}, T^{c}\right\}\right)=0$ where $T^{a, b, c}$ are any of the group generators which stand at the three gauge boson vertices of the triangle diagrams.

${ }^{11}$ We will not include the analysis of the $U(1)_{X}^{3}$ or of the gravitational anomaly because they depend exclusively on SM singlet fields.
} 
family dependent (FD) part of the $U(1)_{X}$ charges.

In this paper we will assume that the cancellation of anomalies results from the Green-Schwartz (GS) mechanism [39]. This is possible if the $A_{3}, A_{2}$ and $A_{1}$ anomalies are in the ratio $A_{3}: A_{2}: A_{1}=k_{3}: k_{2}: k_{1}$ where the $k_{i}$ are the Kac-Moody levels of the $S U(3), S U(2)$ and $U(1)_{Y}$ gauge groups that determine the boundary conditions for the gauge couplings at the string scale $g_{3}^{2} k_{3}: g_{2}^{2} k_{2}: g_{1}^{2} k_{1}$. Hence, using the canonical GUT normalization for the gauge couplings (that successfully predicts $\sin ^{2}\left(\theta_{W}\right)=3 / 8$ [40]), anomalies can be cancelled if we require that:

$$
A_{3}=A_{2}=\frac{5}{3} A_{1}
$$

As a consequence of the two constraints implicit in Eq. (46), the set of solutions for the $X$ charges appearing in Eqs. (42)-(44) is given by [32] :

$$
\begin{array}{lll}
X_{e^{c}}=\sum_{A=1}^{3} X_{e_{A}^{c}}=x & X_{l^{c}}=\sum_{A=1}^{3} X_{l_{A}}=y & X_{h_{u}}=-z \\
X_{q}=\sum_{A=1}^{3} X_{q_{A}}=x+u & X_{d^{c}}=\sum_{A=1}^{3} X_{d_{A}^{c}}=y+v & X_{h_{d}}=+z+(u+v) \\
X_{u^{c}}=\sum_{A=1}^{3} X_{u_{A}^{c}}=x+2 u & &
\end{array}
$$

where $x, y, z, u, v$ are free parameters. However not all the solutions in Eqs. (47) are valid after $A_{1}^{\prime}=0$ is enforced. In fact, as we said before, generally $A_{1}^{\prime}$ constrains both the FI and FD charges of $U(1)_{X}$. By this we mean that, if we conveniently write the charge of the $f_{A}$ field $X_{f_{A}}$ as a sum of a FI part $X_{f}$ plus a FD part $X_{f_{A}}^{\prime}$, i.e. $X_{f_{A}}=\frac{1}{3} X_{f}+X_{f_{A}}^{\prime}$, then $A_{1}^{\prime}=0$ is a complicated equation on all $X_{f}, X_{f_{A}}^{\prime}$ and $X_{h_{u}}$, $X_{h_{d}}$ charges. However, it is easy to see that, if all the left-handed fields and if all the right-handed fields have the same FD charges, i.e. $X_{q_{A}}^{\prime}=X_{l_{A}}^{\prime}$ and $X_{u_{A}^{c}}^{\prime}=X_{d_{A}^{c}}^{\prime}=X_{e_{A}^{c}}^{\prime}$, as is the case of the 422 model, then $A_{1}^{\prime}=0$ is an equation on the FI charges only :

$$
A_{1}^{\prime}=\frac{2}{3}\left(X_{q}^{2}-2 X_{u^{c}}^{2}+X_{d^{c}}^{2}-X_{l}^{2}+X_{e^{c}}^{2}+X_{h_{u}}^{2}-X_{h_{d}}^{2}\right)=0
$$

Thus, a simple solution to all the anomaly constraints is given by Eq. (47) with $u=$ $v=0$. Finally, we must add that since the Pati-Salam model unifies all the left/right- 
handed quark and lepton fields in the $F / F^{c}$ multiplets, and the MSSM Higgs fields $h_{u}, h_{d}$ in the $h$ Higgs bi-doublet we must also have $x=y$ and $z=0$. Thus, anomaly cancellation in the 422 model via GS mechanism is possible if the traces of the $U(1)_{X}$ charges of the $F$ and $F^{c}$ fields are equal, ie $X_{F}=\sum_{A=1}^{3} X_{F_{A}} \equiv \sum_{A=1}^{3} X_{F_{A}^{c}}=X_{F^{c}}$.

\section{OPERATOR APPROACH TO FERMION MASSES}

In the simplest formulation of the 422 model extended by a $U(1)_{X}$ horizontal symmetry all the Yukawa couplings originate from a single matrix. The Abelian $U(1)_{X}$ symmetry introduced in the previous section mainly serves one purpose, it establishes an hierarchy between the flavour dependent couplings. Thus, it provides no precise/predictive information about the relationships between the different Yukawa coupling matrices. As a result, all the SM fermions of a given family have identical Yukawa couplings at the unification scale. Naturally, when the fermion masses are run from the $M_{X}$ to the $M_{Z}$ scale they lead to quark and lepton masses that are incompatible with the experimental data.

The idea of Yukawa unification, though unsuccessful in its most simpler form, is not, however, a complete failure. As a matter of fact, it turns out that third family Yukawa unification works rather well. It is well known that the GUT boundary condition for the Yukawa couplings :

$$
\lambda_{t}\left(M_{X}\right)=\lambda_{b}\left(M_{X}\right)=\lambda_{\tau}\left(M_{X}\right)=\lambda_{\nu_{\tau}}\left(M_{X}\right)
$$

leads to a large pole top mass prediction $M_{t} \sim 175 \mathrm{GeV}$ and $\tan \beta \sim m_{t} / m_{b}$. On the other hand, the first and second family fermion masses can be predicted if special relations between the "vertical" intra-generation Yukawa couplings at $M_{X}$ hold. For example, the Georgi-Jarlskog (GJ) 41] relation between the muon and strange Yukawa couplings $\lambda_{\mu} \sim 3 \lambda_{s}$ successfully reproduces the low energy experimental $m_{s} / m_{\mu} \sim 1$ mass ratio. In the context of GUT theories the appearance of numerical 
factors relating the couplings of the up-down-lepton Yukawa matrices might originate from non-renormalizable operators involving the interaction between the fermions and the heavy Higgs that break the GUT symmetry [42, 43].

In the Pati-Salam model, we will have in mind operators of the following form [24]:

$$
F_{A} F_{B}^{c} h\left(\frac{H \bar{H}}{M_{V}^{2}}\right)^{n}\left(\frac{\theta}{M_{V}}\right)^{p_{A B}} \quad \text { and } \quad F_{A} F_{B}^{c} h\left(\frac{H \bar{H}}{M_{V}^{2}}\right)^{n}\left(\frac{\bar{\theta}}{M_{V}}\right)^{p_{A B}}
$$

The idea is that when the $H$ and $\theta$ fields develop their VEVs such operators reduce to effective Yukawa couplings with small coefficients. For example, if $F_{2}, F_{2}^{c}$ and $h$ carry a charge $X_{F_{2}}=0, X_{F_{2}^{c}}=2$ and $X_{h}=0$ under the $U(1)_{X}$ symmetry then Eq. (50) (with $n=1$ ) generates the following terms :

$$
\left(x_{u} u_{2} u_{2}^{c} h_{u}^{0}+x_{d} d_{2} d_{2}^{c} h_{d}^{0}+x_{e} e_{2} e_{2}^{c} h_{d}^{0}+x_{\nu} \nu_{2} \nu_{2}^{c} h_{u}^{0}\right) \delta \epsilon^{2}
$$

where $\delta=\langle H\rangle\langle\bar{H}\rangle / M_{V}^{2}$ and $\epsilon=\langle\theta\rangle / M_{V}$ are small dimensionless parameters, $u_{2}, d_{2}$, $e_{2}, \nu_{2}$ are the charm, strange, muon, muon neutrino superfields, and $x_{f}(f=u, d, e, \nu)$ are Clebsch factors that depend on the group theoretical contractions between the fields in Eq. (50) [21, 22]. In Table XI (Appendix A) we present a complete list of all $x_{f}$ values that result from $n=1$ operators in the 422 model [24] normalized by :

$$
x_{u}^{2}+x_{d}^{2}+x_{e}^{2}+x_{\nu}^{2}=4
$$

It is interesting to point out that different operators imply zero Clebsches for different $x_{f}$ 's. For example, CLASS-I operators are rather special since of all $x_{f}$ 's only one is non-zero (and significantly large). The CLASS-II operators have $x_{u}=x_{\nu}=0$ while CLASS-III have $x_{d}=x_{e}=0$. Additionally CLASS-IV operators have $x_{u}=x_{d}=0$ and CLASS-V have $x_{e}=x_{\nu}=0$. Finally CLASS-VI operators have all $x_{f}$ 's different from zero. The variety of the operator Clebsches is to be welcome since, as we will see, they open the possibility of avoiding the disastrous fermion mass predictions characteristic of the minimal 422 model with a unified renormalizable interaction. 
Finally we shall mention the origin of the heavy Majorana neutrino mass matrix. Generally $M_{R R}$ results from non-renormalizable operators of the form :

$$
F_{A}^{c} F_{B}^{c}\left(\frac{H H}{M_{V}^{2}}\right)\left(\frac{H \bar{H}}{M_{V}^{2}}\right)^{n}\left(\frac{\theta}{M_{V}}\right)^{q_{A B}} \rightarrow \nu_{A}^{c} \nu_{B}^{c} \delta^{n+1} \epsilon^{q_{A B}}
$$

where $q_{A B}=\left|X_{F_{A}^{c}}+X_{F_{B}^{c}}+\sigma\right|$ and $\sigma=2 X_{H}$. Three important differences distinguish Eq. (53) from Eq. (50). Firstly we note that while Eq. (50) allows for renormalizable operators, $M_{R R}$ as given by Eq. (53) is always the result of non-renormalizable operators. Secondly we note that the combination of the $H H$ fields in Eq. (53) introduces an additional free parameter $\sigma$ that may be fixed at our convenience. Thirdly we observe that while Eq. (50) is able to generate precise relationships between the updown-lepton Yukawa couplings (via Clebsch factors), Eq. (53) is an expression that constrains only the hierarchy of $M_{R R}$ (via the $U(1)_{X}$ symmetry), as a result it is less predictive.

\section{NEUTRINO MASSES AND MIXING ANGLES}

In this section we show how the $U(1)_{X}$ horizontal family symmetry of section $\mathrm{V}$ can be combined with the operator approach of section VI to give predictions for the fermion masses and mixing angles in the 422 model. In particular we are interested in the predictions for the neutrino masses and mixing angles for the LMA MSW solution to the solar neutrino problem. The LOW and the SMA MSW solutions are discussed in Appendices C and D. We start by listing the quark and charged lepton experimental data used in our analysis : एँ

$$
\begin{array}{rlc}
m_{u}(1 \mathrm{GeV})=4.7 \mathrm{MeV} & (1.35-6.75) \mathrm{MeV} \\
m_{c}\left(M_{c}\right)=1.21 \mathrm{GeV} & (1.15-1.35) \mathrm{GeV} \\
M_{t} \sim 175 \mathrm{GeV} & (170-180) \mathrm{GeV}
\end{array}
$$

\footnotetext{
${ }^{12}$ The numbers inside the curly brackets indicate the experimental ranges according to Ref. [44].
} 


$$
\begin{array}{ccc}
m_{d}(1 \mathrm{GeV}) & \sim 6.0 \mathrm{MeV} & (4-12) \mathrm{MeV} \\
m_{s}\left(M_{s}\right) & \sim 160 \mathrm{MeV} \quad(100-230) \mathrm{MeV} \\
m_{b}\left(M_{b}\right)=4.15 \mathrm{GeV} & (4.0-4.4) \mathrm{GeV} \\
M_{e}=0.511 \mathrm{MeV} \quad m_{e}\left(M_{e}\right)=0.496 \mathrm{MeV} \\
M_{\mu}=105.7 \mathrm{MeV} \quad m_{\mu}\left(M_{\mu}\right)=104.6 \mathrm{MeV} \\
M_{\tau}=1777.0 \mathrm{MeV} \quad m_{\tau}\left(M_{\tau}\right)=1772.8 \mathrm{MeV}
\end{array}
$$

where $m_{u}(1 \mathrm{GeV}), m_{d}(1 \mathrm{GeV})$ denote the running masses of the up and down quarks at $Q=1 \mathrm{GeV}$; $m_{c}\left(M_{c}\right), m_{s}\left(M_{c}\right), m_{b}\left(M_{b}\right)$ the running masses of the charm, strange and bottom quarks at their pole masses $\left(M_{c}=1.6 \mathrm{GeV}, M_{b}=4.8 \mathrm{GeV}\right) ; M_{t}$ the top pole mass; and $M_{e, \mu, \tau}\left(m_{e, \mu, \tau}\right)$ the well known pole (running) charged lepton masses. We converted the above pole masses to running masses using the expressions in Ref. [45] with :

$$
\alpha_{s}\left(M_{Z}\right)=0.120 \quad \alpha_{e}^{-1}\left(M_{Z}\right)=127.8
$$

Finally the CKM matrix at $Q=M_{Z}$ was fixed by : "

$$
\left|V_{12}\right|=0.2215 \quad\left|V_{23}\right|=0.040 \quad\left|V_{13}\right|=0.0035
$$

It is important to note that, in fact, not all the parameters above were taken as an input. Indeed, $M_{t} \sim 175 \mathrm{GeV}$ is a prediction that results from third family Yukawa unification $\lambda_{t}=\lambda_{b}=\lambda_{\tau}$ at the GUT scale. Moreover, as we will see, our model is also able to predict the masses of the down and charm quarks, thus their values listed above should be taken merely as a guide and/or convenient initial estimates.

We now turn to the neutrino experimental data. The results from the SuperKamiokande collaboration [1, 2] indicate that the atmospheric neutrino anomaly can

\footnotetext{
${ }^{13}$ All running masses are given in the $\overline{\mathrm{MS}}$ scheme.

14 The experimental ranges are $\left|V_{12}\right|=0.219$ to $0.226,\left|V_{23}\right|=0.037$ to 0.043 and $\left|V_{13}\right|=$ 0.002 to 0.005 [44].
} 
be understood in terms of $\nu_{\mu} \leftrightarrow \nu_{\tau}$ oscillations with :

$$
\sin ^{2}\left(2 \theta_{23}\right)>0.88 \quad 1.5 \times 10^{-3} \mathrm{eV}^{2}<\Delta m_{23}^{2}<5 \times 10^{-3} \mathrm{eV}^{2}
$$

at $90 \%$ confidence level. On the other hand, the large mixing angle (LMA) MSW solution to the solar neutrino deficit suggests that [5] :

$$
\sin ^{2}\left(2 \theta_{12}\right) \sim 0.75 \quad \Delta m_{12}^{2} \sim 2.5 \times 10^{-5} \mathrm{eV}^{2}
$$

Assuming that the neutrino spectrum is hierarchical, i.e. $\Delta m_{23}^{2}=\left|m_{\nu_{3}}^{2}-m_{\nu_{2}}^{2}\right| \sim m_{\nu_{3}}^{2}$ and $\Delta m_{12}^{2}=\left|m_{\nu_{2}}^{2}-m_{\nu_{1}}^{2}\right| \sim m_{\nu_{2}}^{2}$ the values in Eqs. (65), (66) give :

$$
\sin \left(\theta_{23}\right)>0.57 \quad m_{\nu_{3}} \sim 0.05 \mathrm{eV} \quad \sin \left(\theta_{12}\right) \sim 0.50 \quad m_{\nu_{2}} \sim 0.005 \mathrm{eV}
$$

The latest results from the $\mathrm{CHOOZ}$ experiment also show that, over the interesting $\Delta m_{23}^{2}$ range suggested by the Super-Kamiokande data, $\sin ^{2}\left(2 \theta_{13}\right)<0.10$ at 90\% CL 46].

The experimental data in Eqs. (54)-(64) constrains the parameters of our model at low energy. However, the GUT symmetry is broken at an energy $M_{X} \sim 10^{16} \mathrm{GeV}$. Thus, before we start our analysis we should correct the fermion masses and mixing angles for the radiative corrections that result from the running of the RGEs between the $Q=M_{Z}$ and $Q=M_{X}$ scales. The implementation of the RGEs, decoupling of SUSY particles and boundary conditions is a complicated subject whose detailed description is beyond the scope of this work. Here we will only mention that we used 2-loop RGEs in the gauge and Yukawa couplings and refer the interested reader to Ref. 25] where the issue of Yukawa unification in the 422 model is discussed. As a result of the RGEs running, subjected to third family Yukawa unification at $M_{X}$, the low energy input values for the fermion masses in Eqs. (54)-(62) effectively constraint the eigenvalues of the Yukawa couplings at $Q=M_{X}$ to be :

$$
\lambda_{u}\left(M_{X}\right)=4.738 \times 10^{-6} \quad \lambda_{c}\left(M_{X}\right)=1.529 \times 10^{-3} \quad \lambda_{t}\left(M_{X}\right)=0.677
$$




$$
\begin{array}{lll}
\lambda_{d}\left(M_{X}\right) \sim 3.208 \times 10^{-4} & \lambda_{s}\left(M_{X}\right) \sim 9.612 \times 10^{-3} & \lambda_{b}\left(M_{X}\right)=0.677 \\
\lambda_{e}\left(M_{X}\right)=1.490 \times 10^{-4} & \lambda_{\mu}\left(M_{X}\right)=3.154 \times 10^{-2} & \lambda_{\tau}\left(M_{X}\right)=0.677
\end{array}
$$

and the CKM matrix at $Q=M_{X}$ to be :

$$
\left|V_{12}\left(M_{X}\right)\right|=0.2215 \quad\left|V_{23}\left(M_{X}\right)\right|=0.032 \quad\left|V_{13}\left(M_{X}\right)\right|=0.0028
$$

At this point it is convenient to re-write Eqs. (68)-(71) in terms of the Wolfenstein [37] expansion parameter $\lambda=0.22 \sim\left|V_{12}\right|$. We find :

$$
\begin{aligned}
& \lambda_{u}\left(M_{X}\right)=\lambda^{8.097} \quad \lambda_{c}\left(M_{X}\right)=\lambda^{4.282} \quad \lambda_{t}\left(M_{X}\right)=\lambda^{0.257} \\
& \lambda_{d}\left(M_{X}\right) \sim \lambda^{5.313} \quad \lambda_{s}\left(M_{X}\right) \sim \lambda^{3.068} \quad \lambda_{b}\left(M_{X}\right)=\lambda^{0.257} \\
& \lambda_{e}\left(M_{X}\right)=\lambda^{5.820} \quad \lambda_{\mu}\left(M_{X}\right)=\lambda^{2.283} \quad \lambda_{\tau}\left(M_{X}\right)=\lambda^{0.257} \\
& \left|V_{12}\left(M_{X}\right)\right|=\lambda^{0.996} \quad\left|V_{23}\left(M_{X}\right)\right|=\lambda^{2.273} \quad\left|V_{13}\left(M_{X}\right)\right|=\lambda^{3.882}
\end{aligned}
$$

Equations (72)-(75) neatly summarize the hierarchy of the quark and charged lepton sectors at $M_{X}$ that we aim to reproduce/predict.

It is now time to specify the structure of the LMA model in more detail. We start by indicating the nature of the (non-)renormalizable operators responsible for the structure of the Dirac and neutrino Majorana matrices :

$$
\begin{aligned}
\lambda_{A B}: & F_{3} F_{3}^{c} h+F_{A} F_{B}^{c} h \frac{H \bar{H}}{M_{V}^{2}}\left[1+\left(\frac{H \bar{H}}{M_{V}^{2}}\right)+\left(\frac{H \bar{H}}{M_{V}^{2}}\right)^{2}+\ldots\right]\left(\frac{\theta}{M_{V}}\right)^{p_{A B}} \\
\left(M_{R R}\right)_{A B}: & \left\{F_{3}^{c} F_{3}^{c}+F_{A}^{c} F_{B}^{c}\left[\frac{H \bar{H}}{M_{V}^{2}}+\ldots\right]\right\} \frac{H H}{M_{V}^{2}}\left(\frac{\theta}{M_{V}}\right)^{q_{A B}}
\end{aligned}
$$

The first term in Eq. (76) is renormalizable, thus it implies third family Yukawa unification at $M_{X}$. The second term, which we shall assume to be present for $A B \neq$ 33, on the other hand, is a sum of non-renormalizable operators. For the sake of simplicity we will consider that the $H \bar{H} / M_{V}^{2}$ part of $\lambda_{A B}$ that lies outside the square brackets in Eq. (76) has non-trivial gauge contractions with the $F_{A} F_{B}^{c} h$ fields next to 
it, thereby generating the Clebsch factors in Table XI (Appendix A). On the other hand, the $\left(H \bar{H} / M_{V}^{2}\right)^{1,2}$ factors inside the square brackets will form gauge singlet terms that will be responsible for the appearance of higher $\delta$ powers in the entries of $\lambda_{A B}$. The $M_{R R}$ matrix, as given by Eq. (77), depends only on non-renormalizable operators because gauge invariance demands that every combination of $F^{c} F^{c}$ fields must be paired with at least a couple of $H H$ fields. However, we will assume that the only $n=1$ operator in $M_{R R}$ is placed on the 33 entry. All other entries of $M_{R R}$ result from $n=2$ operators. 四

We can see from Eqs. (76),(77) that the structure of the Yukawa and Majorana matrices can be decomposed into a "vertical" $\delta$-component and a "horizontal" $\epsilon$ component. Thus we write :

$$
\left(\lambda_{f}\right)_{A B} \sim\left(\lambda^{\delta}\right)_{A B}\left(\lambda^{\epsilon}\right)_{A B} \quad\left(M_{R R}\right)_{A B} \sim\left(M_{R R}^{\delta}\right)_{A B}\left(M_{R R}^{\epsilon}\right)_{A B}
$$

The hierarchies of $\lambda^{\epsilon}$ and $M_{R R}^{\epsilon}$ are fixed by the choice of the $U(1)_{X}$ charges. Using the results of Ref. [1], we can write the most general form of the unified $\left(\lambda^{\epsilon}\right)_{A B}$ matrix in the 422 model, constrained by the absence of anomalies, in terms of only four independent parameters $\bar{X}_{F_{1}}, \bar{X}_{F_{2}}, \bar{X}_{F_{1}^{c}}$ and $\bar{X}_{F_{2}^{c}}$ : 四

$$
\lambda^{\epsilon}=\left(\begin{array}{ccc}
\epsilon^{\left|\bar{X}_{F_{1}}+\bar{X}_{F_{1}^{c}}\right|} & \epsilon^{\left|\bar{X}_{F_{1}}+\bar{X}_{F_{2}}\right|} & \epsilon^{\left|\bar{X}_{F_{1}}\right|} \\
\epsilon^{\left|\bar{X}_{F_{2}}+\bar{X}_{F_{1}^{c}}\right|} & \epsilon^{\left|\bar{X}_{F_{2}}+\bar{X}_{F_{2}}\right|} & \epsilon^{\left|\bar{X}_{F_{2}}\right|} \\
\epsilon^{\left|\bar{X}_{F_{1}^{c}}\right|} & \epsilon^{\left|\bar{X}_{F_{2}^{c}}\right|} & 1
\end{array}\right)
$$

From the equation above it is easy to see that the values of $\bar{X}_{F_{2}}, \bar{X}_{F_{2}^{c}}, \bar{X}_{F_{1}}$ and $\bar{X}_{F_{1}^{c}}$ are closely related with the large neutrino $\theta_{23}$ angle, the second family Yukawa couplings, the $V_{12} \mathrm{CKM}$ angle, and the masses of the lightest fermions respectively. In the first row of Table I we list our choices for the $\bar{X}$ 's parameters which we will, from now on, refer to as $U(1)_{\bar{X}}$ charges. In the second row we indicate the values of the physical

\footnotetext{
${ }^{15}$ We note that these assumptions about the nature of the Majorana matrix are unique to the LMA MSW solution. The SMA MSW and LOW solutions discussed in Appendices C and D are characterized by a Majorana matrix filled with $n=1$ operators only.

${ }^{16}$ In [11] these charges were called $\alpha, \beta, \gamma, \delta$. Roughly, this corresponds to choosing a basis of charges, that has $\bar{X}_{F_{3}}=\bar{X}_{F_{3}^{c}}=\bar{X}_{h}=0$.
} 
(anomaly free) $U(1)_{X}=U(1)_{F D}+U(1)_{F I}$ charges of the fields of our model. In the third and forth row we list the values of the family dependent (traceless) and family independent (unphysical) charges that sum up to give $U(1)_{X}$.

TABLE I

\begin{tabular}{lccrrrrrrrr}
\hline \hline & & $X_{F_{1}}$ & $X_{F_{2}}$ & $X_{F_{3}}$ & $X_{F_{1}^{c}}$ & $X_{F_{2}^{c}}$ & $X_{F_{3}^{c}}$ & $X_{h}$ & $X_{H}$ & $X_{\bar{H}}$ \\
\hline$U(1)_{\bar{X}}$ & $:$ & 1 & 0 & 0 & 4 & 2 & 0 & 0 & 0 & 0 \\
$U(1)_{X}$ & $:$ & $\frac{11}{6}$ & $\frac{5}{6}$ & $\frac{5}{6}$ & $\frac{19}{6}$ & $\frac{7}{6}$ & $-\frac{5}{6}$ & 0 & $\frac{5}{6}$ & $-\frac{5}{6}$ \\
$U(1)_{F D}$ & $:$ & $\frac{2}{3}$ & $-\frac{1}{3}$ & $-\frac{1}{3}$ & 2 & 0 & -2 & $\frac{7}{3}$ & 2 & -2 \\
$U(1)_{F I}$ & $:$ & $\frac{7}{6}$ & $\frac{7}{6}$ & $\frac{7}{6}$ & $\frac{7}{6}$ & $\frac{7}{6}$ & $\frac{7}{6}$ & $-\frac{7}{3}$ & $-\frac{7}{6}$ & $\frac{7}{6}$ \\
\hline \hline
\end{tabular}

TABLE I. List of $U(1)$ flavour charges that determine the family structure of the Yukawa and neutrino Majorana matrices of the LMA model. The first set, indicated by $U(1)_{\bar{X}}$, refers to the values of the $\bar{X}$ parameters that determine the hierarchy of $\lambda^{\epsilon}$ in Eq. (79). The second set, $U(1)_{X}=U(1)_{F D}+U(1)_{F I}$, corresponds to the anomaly free physical flavour charges of the model. The third set $U(1)_{F D}$ indicates the charges of the family dependent (traceless) component of $U(1)_{X}$ and $U(1)_{F I}$ refers to the family independent component of $U(1)_{X}$.

We note that the $U(1)_{\bar{X}}$ and $U(1)_{X}$ charges are "equivalent" in the sense that they determine equal family structures for the Yukawa and neutrino Majorana matrices. ए

The charges in Table I fix the $\epsilon$-structure of $\lambda^{\epsilon}$ and $M_{R R}^{\epsilon}$ to be :

$$
\lambda^{\epsilon} \sim\left(\begin{array}{ccc}
\epsilon^{5} & \epsilon^{3} & \epsilon \\
\epsilon^{4} & \epsilon^{2} & 1 \\
\epsilon^{4} & \epsilon^{2} & 1
\end{array}\right) \quad M_{R R}^{\epsilon} \sim\left(\begin{array}{ccc}
\epsilon^{8} & \epsilon^{6} & \epsilon^{4} \\
\epsilon^{6} & \epsilon^{4} & \epsilon^{2} \\
\epsilon^{4} & \epsilon^{2} & 1
\end{array}\right)
$$

Comparing $\lambda^{\epsilon}$ above with the hierarchy of the Yukawa couplings listed in Eqs. (72)(75) we see that, the $U(1)_{X}$ symmetry (by itself) can not explain the pattern of all fermion masses and mixing angles. For example, although the symmetry allows a large 23 entry suitable for generating large 23 mixing from the neutrino Yukawa matrix, it also allows similarly large 23 entries in the charged lepton and quark Yukawa matrices which are not welcome. In order to overcome this we shall assume that although a renormalizable operator in the 23 position is allowed by the $U(1)_{X}$ symmetry, it is

\footnotetext{
${ }^{17}$ However, only the $U(1)_{X}$ symmetry is anomaly free.
} 
forbidden by some unspecified string symmetry which however allows a 23 operator containing one factor of $(H \bar{H})$. We shall further select a 23 operator which will involve a Clebsch factor of zero for the charged lepton and quark entries, with only its neutrino component having a non-zero contribution, thereby generating a large 23 mixing from the neutrino sector, with only small 23 mixing in the charged lepton and quark sectors arising from operators containing higher powers of $(H \bar{H})^{n}$, with $n>1$. The existence of such operators with "Clebsch zeros" is clearly crucial for the success of our approach.

In general, we shall show that by a suitable choice of non-renormalizable operators, which determine the $\lambda^{\delta}$ "vertical" structure of $\lambda_{f}$, we can obtain a successful description of all quark and lepton masses and mixing angles. For example, let us consider $\lambda^{\delta}$ and $M_{R R}^{\delta}$ given by the following operator matrices :

$$
\lambda^{\delta} \sim\left(\begin{array}{ccc}
\mathcal{O}^{R}+\mathcal{O}^{\prime \prime V} & \mathcal{O}^{J}+\mathcal{O}^{\prime Q} & \mathcal{O}^{g}+\mathcal{O}^{\prime f} \\
\mathcal{O}^{G}+\mathcal{O}^{\prime \prime} & \mathcal{O}^{W}+\mathcal{O}^{\prime H} & \mathcal{O}^{I}+\mathcal{O}^{\prime W} \\
\mathcal{O}^{R}+\mathcal{O}^{\prime \prime V} & \mathcal{O}^{M}+\mathcal{O}^{\prime K} & 1
\end{array}\right) \quad M_{R R}^{\delta} \sim\left(\begin{array}{ccc}
\mathcal{O} & \mathcal{O} & \mathcal{O} \\
\mathcal{O} & \mathcal{O} & \mathcal{O} \\
\mathcal{O} & \mathcal{O} & 1
\end{array}\right)
$$

where $\mathcal{O}, \mathcal{O}^{\prime}$ and $\mathcal{O}^{\prime \prime}$ are $n=1, n=2$ and (very small) $n=3$ operators respectively where $n$ is defined in Eq. (50) and refers to the powers of $(H \bar{H})^{n}$. Using Eqs. (80), (81) into Eq. (78) gives :

$$
\begin{aligned}
\lambda_{f}\left(M_{X}\right)= & \left(\begin{array}{ccc}
x_{f}^{R} a_{11} \delta \epsilon^{5} & x_{f}^{J} a_{12} \delta \epsilon^{3} & x_{f}^{g} a_{13} \delta \epsilon^{2} \\
x_{f}^{G} a_{21} \delta \epsilon^{4} & x_{f}^{W} a_{22} \delta \epsilon^{2} & x_{f}^{I} a_{23} \delta \\
x_{f}^{R} a_{31} \delta \epsilon^{4} & x_{f}^{M} a_{32} \delta \epsilon^{2} & a_{33}
\end{array}\right)+ \\
& \left(\begin{array}{ccc}
0 & x_{f}^{Q} a_{12}^{\prime} \delta^{2} \epsilon^{3} & x_{f}^{f} a_{13}^{\prime} \delta^{2} \epsilon^{2} \\
0 & x_{f}^{H} a_{22}^{\prime} \delta^{2} \epsilon^{2} & x_{f}^{W} a_{23}^{\prime} \delta^{2} \\
0 & x_{f}^{K} a_{32}^{\prime} \delta^{2} \epsilon^{2} & 0
\end{array}\right)+ \\
& \left(\begin{array}{ccc}
x_{f}^{V} a_{11}^{\prime \prime} \delta^{3} \epsilon^{5} & 0 & 0 \\
x_{f}^{K} a_{21}^{\prime \prime} \delta^{3} \epsilon^{4} & 0 & 0 \\
x_{f}^{V} a_{23}^{\prime \prime} \delta^{3} \epsilon^{4} & 0 & 0
\end{array}\right) \\
\frac{M_{R R}\left(M_{X}\right)}{M_{R R}\left(M_{X}\right)_{33}}= & \left(\begin{array}{lll}
A_{11} \delta \epsilon^{8} & A_{12} \delta \epsilon^{6} & A_{13} \delta \epsilon^{4} \\
A_{21} \delta \epsilon^{6} & A_{22} \delta \epsilon^{4} & A_{23} \delta \epsilon^{2} \\
A_{31} \delta \epsilon^{4} & A_{32} \delta \epsilon^{2} & A_{33}
\end{array}\right)
\end{aligned}
$$

\footnotetext{
${ }^{18}$ The $n=3$ operators can, to a very good approximation, be neglected. Their inclusion here serves only to fill the 11, 21, 31 entries of the $\lambda_{u, \nu}$ Yukawa matrices, thereby ensuring (for example) that the up quark is given a very small mass.
} 
where the subscript $f$ stands for any of the $u, d, e, \nu$ indices, $x_{f}^{\mathcal{O}}$ is the Clebsch of the $\mathcal{O}$ operator of the $f$-type fermion, and the $a$ 's $(A$ 's) are order-one $f$-independent Yukawa (Majorana) parameters that parameterise $\lambda_{f}\left(M_{R R}\right)$. The first matrix on the right-hand side of Eq.82 contains the leading $n=1$ operators giving contributions of order $\delta$, while the second and third matrices contain the $n=2$ and $n=3$ operators which give contributions of order $\delta^{2}$ and $\delta^{3}$ and provide the leading contributions in the cases where the $n=1$ operators involve Clebsch zeros.

The effective matrices resulting from Eqs. (82), (83) are approximately given in Table II. This table shows an interesting structure for the Yukawa matrices. We find that $\lambda_{u} \sim \lambda^{8}, \lambda_{c} \sim \lambda^{4}$ and $\lambda_{d} \sim \lambda_{e} \sim \lambda^{6}, \lambda_{s} \sim \lambda_{\mu} \sim \lambda^{3}$. Furthermore the CKM matrix has $\left|V_{12}\right| \sim \lambda,\left|V_{23}\right| \sim \lambda^{2},\left|V_{13}\right| \sim \lambda^{3}$. Comparing these approximate results with the data in Eqs. (72)-(75) we see that only the $\lambda_{d}, \lambda_{\mu}$ couplings need substantial (one $\lambda$-power) corrections. On the other hand, the neutrino sector described by $\lambda_{\nu}$ and $M_{R R}$ in Table II is clearly dominated by the right-handed tau neutrino and predicts $\left(\lambda_{\nu}\right)_{12} \sim\left(\lambda_{\nu}\right)_{22}$ which according to Eq. (34) successfully generates a large $\theta_{12}$ solar neutrino angle. However, the subdominant perturbation to $m_{L L}$ in Eq. (8) resulting from $\lambda_{\nu}$ and $M_{R R}$ in Table II is too small to correctly predict the neutrino mass ratio $m_{\nu_{2}} / m_{\nu_{3}} \sim \lambda^{1.5}$ required by Eq. (67). These approximate predictions can be further improved because Table II does not include the numerical effects of the operator Clebsches and of the order-one $a, A$ factors. 


\section{TABLE II}

\begin{tabular}{|c|c|c|c|c|c|c|c|c|}
\hline \multirow{3}{*}{$\lambda_{u}\left(M_{X}\right)$} & \multirow{3}{*}{$\sim$} & $\left(\delta^{3} \epsilon^{5}\right.$ & $\delta^{2} \epsilon^{3}$ & $\delta^{2} \epsilon$ & \multirow{3}{*}{$\sim$} & $\left(\lambda^{8}\right.$ & $\lambda^{5}$ & $\left.\lambda^{3}\right)$ \\
\hline & & $\delta^{3} \epsilon^{4}$ & $\delta^{2} \epsilon^{2}$ & $\delta^{3}$ & & $\lambda^{7}$ & $\lambda^{4}$ & $\lambda^{3}$ \\
\hline & & $\delta^{3} \epsilon^{4}$ & $\delta^{2} \epsilon^{2}$ & 1 & & $\lambda^{7}$ & $\lambda^{4}$ & 1 \\
\hline \multirow{3}{*}{$\lambda_{d}\left(M_{X}\right)$} & \multirow{3}{*}{$\sim$} & $\delta \epsilon^{5}$ & $\delta^{2} \epsilon^{3}$ & $\delta^{2} \epsilon$ & \multirow{3}{*}{$\sim$} & $\left(\lambda^{6}\right.$ & $\lambda^{5}$ & $\left.\lambda^{3}\right)$ \\
\hline & & $\delta \epsilon^{4}$ & $\delta \epsilon^{2}$ & $\delta^{2}$ & & $\lambda^{5}$ & $\lambda^{3}$ & $\lambda^{2}$ \\
\hline & & $\delta \epsilon^{4}$ & $\delta \epsilon^{2}$ & 1 & & $\lambda^{5}$ & $\lambda^{3}$ & 1 \\
\hline \multirow{3}{*}{$\lambda_{e}\left(M_{X}\right)$} & \multirow{3}{*}{$\sim$} & $\delta \delta \epsilon^{5}$ & $\delta \epsilon^{3}$ & $\delta \epsilon$ & \multirow{3}{*}{$\sim$} & $\left(\lambda^{6}\right.$ & $\lambda^{4}$ & $\left.\lambda^{2}\right)$ \\
\hline & & $\delta \epsilon^{4}$ & $\delta \epsilon^{2}$ & $\delta^{2}$ & & $\lambda^{5}$ & $\lambda^{3}$ & $\lambda^{2}$ \\
\hline & & $\delta \epsilon^{4}$ & $\delta \epsilon^{2}$ & 1 & & $\lambda^{5}$ & $\lambda^{3}$ & 1 \\
\hline \multirow{3}{*}{$\lambda_{\nu}\left(M_{X}\right)$} & \multirow{3}{*}{$\sim$} & $\left(\delta^{3} \epsilon^{5}\right.$ & $\delta \epsilon^{3}$ & $\delta \epsilon$ & \multirow{3}{*}{$\sim$} & $\left(\lambda^{8}\right.$ & $\lambda^{4}$ & $\lambda^{2}$ \\
\hline & & $\delta^{3} \epsilon^{4}$ & $\delta^{2} \epsilon^{2}$ & $\delta$ & & $\lambda^{7}$ & $\lambda^{4}$ & $\lambda$ \\
\hline & & $\delta^{3} \epsilon^{4}$ & $\delta^{2} \epsilon^{2}$ & 1 & & $\lambda^{7}$ & $\lambda^{4}$ & 1 \\
\hline \multirow{3}{*}{$M_{R R}\left(M_{X}\right)$} & \multirow{3}{*}{$\sim$} & $\left(\delta \epsilon^{8}\right.$ & $\delta \epsilon^{6}$ & $\delta \epsilon^{4}$ & \multirow{3}{*}{$\sim$} & $\left(\lambda^{9}\right.$ & $\lambda^{7}$ & $\lambda^{5}$ \\
\hline & & $\delta \epsilon^{6}$ & $\delta \epsilon^{4}$ & $\delta \epsilon^{2}$ & & $\lambda^{7}$ & $\lambda^{5}$ & $\lambda^{3}$ \\
\hline & & $\delta \epsilon^{4}$ & $\delta \epsilon^{2}$ & 1 & & $\left(\lambda^{5}\right.$ & $\lambda^{3}$ & $1)$ \\
\hline
\end{tabular}

TABLE II. Approximate structure of the Yukawa and neutrino Majorana matrices resulting from Eqs. (82), (83) when the numerical effect of the Clebsch and of the order-one $a, A$ parameters is neglected.

The success of our model (in the SM sector) depends on the ability to find suitable solutions for the $a$ 's in Eq. (82) which simultaneously can account for all the hierarchies in Eqs. (72)-(75). Generally we will require that $0.5<\left|a_{A B}\right|,\left|a_{A B}^{\prime}\right|,\left|a_{A B}^{\prime \prime}\right|<2.0$ for all $A, B=1,2,3$. At first, it looks that such solution is trivial since Eq. (82) depends on 16 parameters, $\square$ while Eqs. (72)-(75) is a set of 9 constraints (on the first and second family Yukawa couplings and CKM entries.) However, we should not forget that $\mathcal{O} \gg \mathcal{O}^{\prime} \gg \mathcal{O}^{\prime \prime}$ and that the CKM matrix constrains only on the 12,13 and 23 entries of $\lambda_{f}$. As a consequence, we find that the parameters in Eqs. (72)(75) are mainly sensitive to $a_{22} \leftrightarrow \lambda_{s, \mu}, a_{11} \leftrightarrow \lambda_{d, e}$, an independent combination of $\left(a_{11}^{\prime \prime}, a_{21}^{\prime \prime}, a_{31}^{\prime \prime}\right) \leftrightarrow \lambda_{u}, a_{22}^{\prime} \leftrightarrow \lambda_{c}$ and $a_{12}^{\prime} \leftrightarrow V_{12}, a_{23}^{\prime} \leftrightarrow V_{23}, a_{13}^{\prime} \leftrightarrow V_{13}$, which allows two predictions to be made $-\lambda_{d}$ and $\lambda_{s}$. Thus we fitted the $a_{22}, a_{11}, a_{11}^{\prime \prime}, a_{22}^{\prime}, a_{12}^{\prime}, a_{23}^{\prime}, a_{13}^{\prime}$

\footnotetext{
${ }^{19}$ We note that $a_{33}$ is fixed by quadruple Yukawa unification at $M_{X}$, i.e. $\lambda_{t}=\lambda_{b}=\lambda_{\tau}=\lambda_{\nu_{\tau}}$.
} 
dependence of $\lambda_{f}$ to the $\lambda_{\mu}, \lambda_{e}, \lambda_{u}, \lambda_{c}$ and $V_{12}, V_{23}, V_{13}$ experimental constraints in Eqs. (72)-(75). The results are shown in Table III. ${ }^{27}$

\section{TABLE III}

\begin{aligned} & \hline \hline$=\left(\begin{array}{rrr}-1.285 & 1.000 & 1.000 \\ 1.000 & -1.420 & 1.000 \\ -1.000 & 1.000 & 0.677\end{array}\right) \\ & a^{\prime}=\left(\begin{array}{rrr}0.000 & 0.700 & 1.015 \\ 0.000 & 0.782 & 0.705 \\ 0.000 & -1.000 & 0.000\end{array}\right) \\ & a^{\prime \prime}=\left(\begin{array}{rrr}0.907 & 0.000 & 0.000 \\ 1.000 & 0.000 & 0.000 \\ 1.000 & 0.000 & 0.000\end{array}\right) \\ & A=\left(\begin{array}{rrr}1.071 & 0.733 & 0.653 \\ 0.733 & 1.072 & 0.567 \\ 0.653 & 0.576 & 1.000\end{array}\right) \\ &$\hline \hline\end{aligned}

TABLE III. Numerical values of the order-one $a, A$ parameters that parameterise the Yukawa and neutrino Majorana matrices in Eqs. (82),(83).

Thus, using Eq. (82) with the $a$ 's of Table III and the Clebsch factors of Table XI (Appendix A) we get the numerical results for $\lambda_{f}\left(M_{X}\right)$ shown in Table IV. In Table V we present the results of Table IV expanded in powers of $\delta=\epsilon=\lambda=0.22$.

\footnotetext{
${ }^{20}$ Fixing all other $a$ 's to be one.

${ }^{21}$ The values of the $A$ parameters in Eq. ( $\sqrt{33}$ ) are not constrained by the experimental data, thus we chose them to be "arbitrary" numbers in the $0.5<A_{A B}<2.0$ range.
} 
TABLE IV

\begin{aligned} & \hline \hline$\lambda_{u}\left(M_{X}\right)=\left(\begin{array}{rrc}7.034 \times 10^{-6} & 4.079 \times 10^{-4} & 4.324 \times 10^{-3} \\ 3.991 \times 10^{-5} & 1.466 \times 10^{-3} & 0.000 \\ 3.528 \times 10^{-5} & -3.748 \times 10^{-3} & 0.677\end{array}\right) \\ & \lambda_{d}\left(M_{X}\right)=\left(\begin{array}{rrr}-2.331 \times 10^{-4} & -4.079 \times 10^{-4} & 8.648 \times 10^{-3} \\ 4.609 \times 10^{-4} & -8.827 \times 10^{-3} & 2.157 \times 10^{-2} \\ -8.246 \times 10^{-4} & 1.506 \times 10^{-2} & 0.677\end{array}\right) \\ & \lambda_{e}\left(M_{X}\right)=\left(\begin{array}{rrr}-1.748 \times 10^{-4} & 3.884 \times 10^{-3} & 8.574 \times 10^{-2} \\ 9.219 \times 10^{-4} & 3.015 \times 10^{-2} & -6.472 \times 10^{-2} \\ -6.184 \times 10^{-4} & 1.501 \times 10^{-2} & 0.677\end{array}\right) \\ & \lambda_{\nu}\left(M_{X}\right)=\left(\begin{array}{rrr}7.034 \times 10^{-6} & 2.401 \times 10^{-3} & 7.710 \times 10^{-2} \\ 2.993 \times 10^{-5} & 2.932 \times 10^{-3} & 0.440 \\ 3.528 \times 10^{-5} & -2.811 \times 10^{-3} & 0.677\end{array}\right) \\ & M_{R R}\left(M_{X}\right)=\left(\begin{array}{rrr}3.991 \times 10^{8} & 5.652 \times 10^{9} & 1.040 \times 10^{11} \\ 5.652 \times 10^{9} & 1.706 \times 10^{11} & 1.866 \times 10^{12} \\ 1.040 \times 10^{11} & 1.866 \times 10^{12} & 3.090 \times 10^{14}\end{array}\right) \\ &$\hline\end{aligned}

TABLE IV. Numerical values for the entries of the Yukawa $\left(\lambda_{u}, \lambda_{d}, \lambda_{e}, \lambda_{\nu}\right)$ and neutrino Majorana $\left(M_{R R}\right)$ matrices at the unification scale $M_{X}\left(M_{R R}\right.$ is given in $\mathrm{GeV}$ mass units.)

\section{TABLE V}

\begin{tabular}{|c|c|c|c|c|}
\hline \multirow{3}{*}{$\lambda_{u}\left(M_{X}\right)$} & \multirow{3}{*}{$\sim$} & $\lambda^{7.836}$ & $\lambda^{5.154}$ & $\left.\lambda^{3.595}\right)$ \\
\hline & & $\lambda^{6.690}$ & $\lambda^{4.309}$ & 0 \\
\hline & & $\lambda^{6.771}$ & $-\lambda^{3.690}$ & $\lambda^{0.257}$ \\
\hline \multirow{3}{*}{$\lambda_{d}\left(M_{X}\right)$} & \multirow{3}{*}{$\sim$} & $\left(-\lambda^{5.524}\right.$ & $-\lambda^{5.154}$ & $\left.\lambda^{3.137}\right)$ \\
\hline & & $\lambda^{5.074}$ & $-\lambda^{3.124}$ & $\lambda^{2.534}$ \\
\hline & & $-\lambda^{4.690}$ & $\lambda^{2.771}$ & $\lambda^{0.257}$ \\
\hline \multirow{3}{*}{$\lambda_{e}\left(M_{X}\right)$} & \multirow{3}{*}{$\sim$} & $\left(-\lambda^{5.714}\right.$ & $\lambda^{3.666}$ & $\left.\lambda^{1.622}\right\rangle$ \\
\hline & & $\lambda^{4.616}$ & $\lambda^{2.313}$ & $-\lambda^{1.808}$ \\
\hline & & $-\lambda^{4.880}$ & $\lambda^{2.771}$ & $\lambda^{0.257}$ \\
\hline \multirow{3}{*}{$\lambda_{\nu}\left(M_{X}\right)$} & \multirow{3}{*}{$\sim$} & $\lambda^{7.836}$ & $\lambda^{3.984}$ & $\lambda^{1.693}$ \\
\hline & & $\lambda^{6.880}$ & $\lambda^{3.852}$ & $\lambda^{0.542}$ \\
\hline & & $\lambda^{6.771}$ & $-\lambda^{3.880}$ & $\lambda^{0.257}$ \\
\hline \multirow{3}{*}{$\frac{M_{R R}\left(M_{X}\right)}{M_{R R}\left(M_{X}\right)_{33}}$} & \multirow{3}{*}{$\sim$} & $\lambda^{8.955}$ & $\lambda^{7.205}$ & $\left.\lambda^{5.281}\right)$ \\
\hline & & $\lambda^{7.205}$ & $\lambda^{4.954}$ & $\lambda^{3.375}$ \\
\hline & & $\lambda^{5.281}$ & $\lambda^{3.375}$ & 1 \\
\hline
\end{tabular}

TABLE V. Values for the entries of the Yukawa $\left(\lambda_{u}, \lambda_{d}, \lambda_{e}, \lambda_{\nu}\right)$ and neutrino Majorana $\left(M_{R R}\right)$ matrices at the unification scale $M_{X}$ as given in Table IV but expanded in powers of $\delta=\epsilon=\lambda=0.22\left(\right.$ Note $M_{R R}\left(M_{X}\right)_{33}=3.090 \times 10^{14} \mathrm{GeV}$.) 
We can analyse the effect of the operator Clebsches by comparing Table II against Table V. We see that the $\mathcal{O}^{W}$ operator ${ }^{2}$ in the 22 entry of $\lambda_{f}$ split $\left(\lambda_{d}\right)_{22}=\left(\lambda_{e}\right)_{22} \sim$ $\lambda^{3}$ in Table II into $\left|\left(\lambda_{d}\right)_{22}\right|=\lambda^{3.124}$ and $\left(\lambda_{e}\right)_{22}=\lambda^{2.313}$ in Table V, thus allowing for a proper GJ ratio $\lambda_{\mu} / \lambda_{s} \sim 3$ at $M_{X}$. Similarly, the operators in the 12 block have allowed for a more appropriate $\lambda_{d} / \lambda_{e}$ Yukawa ratio. Numerically we have the following predictions for the lightest eigenvalues of the down-Yukawa matrix at $M_{X}$ $: \lambda_{d}=\lambda^{5.469}$ and $\lambda_{s}=\lambda^{3.087}$.

The effect of the Clebsch factors also modified the neutrino Yukawa matrix $\lambda_{\nu}\left(M_{X}\right)$ in Table II. Due to the $\mathcal{O}^{I}$ operator in the 23 position of $\lambda_{f}$, which has a large $x_{\nu}^{I}=2$ Clebsch, we are now able to predict a large $\theta_{23}$ atmospheric neutrino mixing angle. Indeed using Eq. (19) we can roughly estimate that $\tan \theta_{23} \sim 0.44 / 0.68=0.65$ implying $\sin ^{2}\left(2 \theta_{23}\right) \sim 0.83$.

It is interesting to check that $\lambda_{\nu}$ and $M_{R R}$ in Table $\mathrm{V}$ do lead to a $m_{L L}$ matrix dominated by the right-handed tau neutrino. As a result of the small mixing angles of $M_{R R}$ it is convenient to work in a basis where $M_{R R}$ is diagonal. Furthermore it is practical to scale $\lambda_{\nu}$ s such that the 23 and 33 entries are $\left(\lambda_{\nu}\right)_{23} \sim\left(\lambda_{\nu}\right)_{33} \sim 1$, and approximate the normalized entries to (semi-)integer $\lambda$-powers. Thus using :

$$
M_{R R} \sim\left(\begin{array}{ccc}
\lambda^{9.0} & 0 & 0 \\
0 & \lambda^{5.0} & 0 \\
0 & 0 & 1
\end{array}\right) \quad m_{R L} \sim\left(\begin{array}{ccc}
\lambda^{7.5} & \lambda^{3.5} & \lambda^{1.5} \\
\lambda^{6.5} & \lambda^{3.5} & 1 \\
\lambda^{6.5} & \lambda^{3.5} & 1
\end{array}\right)
$$

into Eq. (8) gives :

$$
m_{L L} \sim\left(\begin{array}{rrr}
\lambda^{3.0}+\lambda^{2.0}+\lambda^{6.0} & \lambda^{1.5}+\lambda^{2.0}+\lambda^{5.0} & \lambda^{1.5}+\lambda^{2.0}+\lambda^{5.0} \\
\lambda^{1.5}+\lambda^{2.0}+\lambda^{5.0} & 1+\lambda^{2.0}+\lambda^{4.0} & 1+\lambda^{2.0}+\lambda^{4.0} \\
\lambda^{1.5}+\lambda^{2.0}+\lambda^{5.0} & 1+\lambda^{2.0}+\lambda^{4.0} & 1+\lambda^{2.0}+\lambda^{4.0}
\end{array}\right)
$$

where the first (second and third) term in each entry corresponds to the third (second and first) family neutrino contribution $\nu_{\tau}^{c}\left(\nu_{\mu}^{c}\right.$ and $\left.\nu_{e}^{c}\right)$ coming from $M_{R R}$. Clearly Eq. (85) shows that even though, in this case, $\nu_{\tau}^{c}$ is the heaviest right-handed neutrino,

\footnotetext{
${ }^{22}$ That has Clebsches $x_{e}^{W}=-3 x_{d}^{W}$

${ }^{23}\left(\lambda_{\nu}\right)_{A B} \rightarrow\left(\lambda_{\nu}\right)_{A B} / k$ with $k=\frac{1}{2}\left[\left(\lambda_{\nu}\right)_{23}+\left(\lambda_{\nu}\right)_{33}\right]=\lambda^{0.384}$.
} 
it nevertheless dominates the 23 block, and that the sub-dominant contribution from $\nu_{\mu}^{c}$ induces $\lambda^{2}$ perturbations in $m_{L L}$ that are compatible with the $m_{\nu_{2}} / m_{\nu_{3}}$ mass ratio.

Using the MSSM RGEs adapted and properly extended to take into account the presence and successive decoupling of the right-handed neutrinos between the $Q=$ $M_{X}$ and $Q=M_{Z}$ scales (see Appendix B) we find that the Yukawa and neutrino Majorana matrices at low energy are given by Table VI.

TABLE VI

\begin{aligned} & \hline \hline$\lambda_{u}\left(M_{Z}\right)=\left(\begin{array}{rrc}1.478 \times 10^{-5} & 8.920 \times 10^{-4} & 5.058 \times 10^{-3} \\ 8.484 \times 10^{-5} & 3.143 \times 10^{-3} & -3.553 \times 10^{-3} \\ 4.687 \times 10^{-5} & -5.015 \times 10^{-3} & 0.905\end{array}\right) \\ & \lambda_{d}\left(M_{Z}\right)=\left(\begin{array}{rrc}-4.605 \times 10^{-4} & -9.389 \times 10^{-4} & 1.175 \times 10^{-2} \\ 9.379 \times 10^{-4} & -1.795 \times 10^{-2} & 3.124 \times 10^{-2} \\ -1.057 \times 10^{-3} & 1.934 \times 10^{-2} & 0.862\end{array}\right) \\ & \lambda_{e}\left(M_{Z}\right)=\left(\begin{array}{rrr}-1.526 \times 10^{-4} & 3.445 \times 10^{-3} & 6.611 \times 10^{-2} \\ 8.832 \times 10^{-4} & 2.931 \times 10^{-2} & -5.446 \times 10^{-2} \\ -4.626 \times 10^{-4} & 1.196 \times 10^{-2} & 0.522\end{array}\right) \\ & \lambda_{\nu}\left(M_{Z}\right)=\left(\begin{array}{ccc}6.110 \times 10^{-6} & 2.271 \times 10^{-3} & 6.339 \times 10^{-2} \\ 2.699 \times 10^{-5} & 2.737 \times 10^{-3} & 0.394 \\ 2.911 \times 10^{-5} & -2.428 \times 10^{-3} & 0.562\end{array}\right) \\ & M_{R R}\left(M_{Z}\right)=\left(\begin{array}{ccc}3.987 \times 10^{8} & 5.651 \times 10^{9} & 9.981 \times 10^{10} \\ 5.651 \times 10^{9} & 1.707 \times 10^{11} & 1.807 \times 10^{12} \\ 9.981 \times 10^{10} & 1.807 \times 10^{12} & 2.879 \times 10^{14}\end{array}\right) \\ &$\hline \hline\end{aligned}

TABLE VI. Numerical values for the entries of the Yukawa $\left(\lambda_{u}, \lambda_{d}, \lambda_{e}, \lambda_{\nu}\right)$ and neutrino Majorana $\left(M_{R R}\right)$ matrices at the $Q=M_{Z}$ scale $\left(M_{R R}\right.$ is given in $\mathrm{GeV}$ mass units.)

Thus, inserting the results of Table VI into Eq. (8) and Eq. (12) we get the mass matrix for the left-handed neutrinos $m_{L L}$ and the $V^{M N S}$ mixing matrix shown in Table VII. The predictions for the neutrino masses and squared mass splittings are shown in Table VIII. In Table IX we examine how the neutrino mixing angles evolve between the unification $Q=M_{X} \sim 3 \times 10^{16} \mathrm{GeV}$, the right-handed tau neutrino mass $Q=M_{\nu_{3}} \sim 3 \times 10^{14} \mathrm{GeV}$ and the $M_{Z}$ scale. We see that the effect of the radiative 
corrections has increased the magnitude of $\sin \theta_{12}, \sin \theta_{23}$ and $\sin \theta_{13}$ by $2.5 \%, 6.4 \%$ and $2.4 \%$ respectively. These corrections agree with the results found in Ref. [47]. Finally, we present in Table $\mathrm{X}$ the predictions for the down and strange quark masses.

We would like to conclude this section by noting that the predictions for the neutrino parameters, in particular for the neutrino $\Delta m_{12}^{2}$ squared mass splitting, should be taken carefully. Generally we expect at least $20 \%$ (theoretical) errors in the quoted values which, for example, arise from our inability to fix order-one factors in the entries of $M_{R R}\left(M_{X}\right)$.

TABLE VII

\begin{tabular}{l}
\hline$\frac{m_{L L}\left(M_{Z}\right)}{m_{L L}\left(M_{Z}\right)_{33}}=\left(\begin{array}{crr}4.792 \times 10^{-2} & 1.007 \times 10^{-1} & 3.458 \times 10^{-2} \\
1.007 \times 10^{-1} & 4.610 \times 10^{-1} & 5.659 \times 10^{-1} \\
3.458 \times 10^{-2} & 5.659 \times 10^{-1} & 1\end{array}\right)$ \\
$V^{M N S}\left(M_{Z}\right)=\left(\begin{array}{rrr}0.8290 & 0.5532 & -0.0819 \\
-0.3948 & 0.6827 & 0.6149 \\
0.3961 & -0.4774 & 0.7843\end{array}\right)$ \\
\hline
\end{tabular}

TABLE VII. Predicted values for the left-handed neutrino mass matrix $m_{L L}\left(M_{Z}\right)$ in units of $m_{L L}\left(M_{Z}\right)_{33}=3.954 \times 10^{-3} \mathrm{eV}$ and for the MNS neutrino mixing matrix $V^{M N S}\left(M_{Z}\right)$.

TABLE VIII

$$
\begin{gathered}
m_{\nu_{1}}=4.84 \times 10^{-8} \mathrm{eV} \quad m_{\nu_{2}}=5.79 \times 10^{-3} \mathrm{eV} \quad m_{\nu_{3}}=5.39 \times 10^{-2} \mathrm{eV} \\
\Delta m_{12}^{2}=3.35 \times 10^{-5} \mathrm{eV}^{2} \quad \Delta m_{23}^{2}=2.87 \times 10^{-3} \mathrm{eV}^{2}
\end{gathered}
$$

TABLE VIII. Predicted values for the left-handed neutrino masses $m_{\nu_{1,2,3}}$ and squared mass splittings $\Delta m_{12}^{2}=\left|m_{\nu_{2}}^{2}-m_{\nu_{1}}^{2}\right|, \Delta m_{23}^{2}=\left|m_{\nu_{3}}^{2}-m_{\nu_{2}}^{2}\right|$. 


\section{TABLE IX}

\begin{tabular}{|c|}
\hline 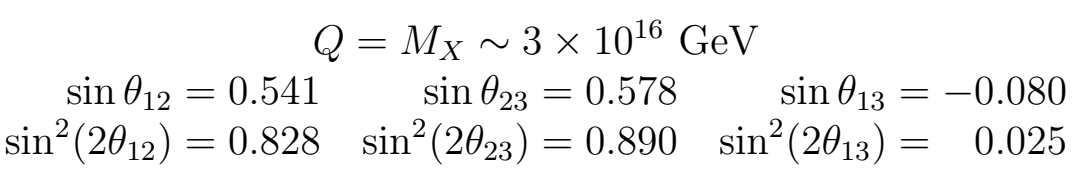 \\
\hline $\begin{array}{ccr}Q=M_{\nu_{3}} \sim 3 \times 10^{14} \mathrm{GeV} \\
\sin \theta_{12}=0.543 \quad \sin \theta_{23}=0.590 & \sin \theta_{13}=-0.082 \\
\sin ^{2}\left(2 \theta_{12}\right)=0.832 & \sin ^{2}\left(2 \theta_{23}\right)=0.908 & \sin ^{2}\left(2 \theta_{13}\right)=0.027\end{array}$ \\
\hline 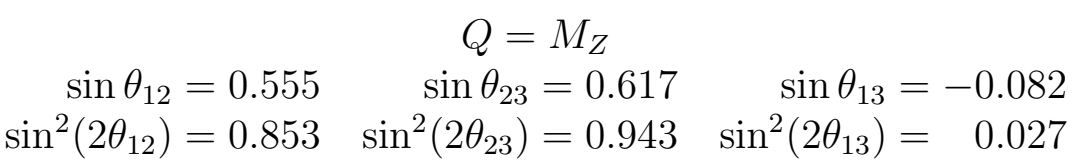 \\
\hline
\end{tabular}

TABLE IX. Running of the neutrino mixing angles at the unification $Q=M_{X}$, the right-handed tau neutrino mass $Q=M_{\nu_{3}}$ and $Z$ boson mass $Q=M_{Z}$ energy scales.

\section{TABLE X}

$\overline{\overline{m_{d}(1 \mathrm{GeV})=4.9 \mathrm{MeV} \quad m_{s}\left(M_{s}\right)=156 \mathrm{MeV}}}$

TABLE X. Predictions for the running $\overline{\mathrm{MS}}$ masses of the down $\left(m_{d}\right)$ and strange $\left(m_{s}\right)$ quarks at $Q=1 \mathrm{GeV}$ and $Q=M_{s}$ respectively $\left(M_{s}\right.$ indicates the strange pole quark mass.) 


\section{CONCLUSION}

We have discussed a theory of all fermion masses and mixing angles based on a particular string-inspired minimal model based on the Pati-Salam group $S U(4) \times$ $S U(2)_{L} \times S U(2)_{R}$ [18 supplemented by a gauged $U(1)_{X}$ family symmetry. We argued that this gauge group preserves the attractive features of $S O(10)$ such as predicting three right-handed neutrinos, and Yukawa unification, while avoiding the doublettriplet splitting problem. Although it is not a unified gauge group at the field theory level, it naturally arises from string constructions and so in principle may be fully unified with gravity.

Earlier work in collaboration with one of us [24 had already shown that the model can provide a successful description of the charged fermion masses and the CKM matrix. The use of the $U(1)_{X}$ family symmetry to provide the horizontal mass splittings combined with the Clebsch factors arising from the $(H \bar{H})^{n}$ insertion in the operators has already been shown to provide a powerful approach to the fermion mass spectrum in this model [24]. The present analysis differs from that presented previously partly due to the recent refinements in third family Yukawa unification [25], but mainly due to the recent data from Super-Kamiokande which implies that the 23 operator should be allowed by the $U(1)_{X}$ family symmetry. We have therefore extended our previous analysis to the atmospheric and solar neutrino masses and mixing angles, and showed that all three MSW solutions to the solar neutrino data may be accommodated, namely the LMA MSW region discussed in the main text as well as the LOW MSW and the SMA MSW regions discussed in the Appendices.

The approach to neutrino masses and mixing angles followed here makes use of the

SRHND mechanism [10, 11, 12] in which one of the right-handed neutrinos (the $\left.\nu_{\tau}^{c}\right)$ gives the dominant contribution to the 23 block of the light effective Majorana matrix. This mechanism avoids reliance on accidental cancellations, and does not rely on 
excessive magnification of mixing angles, although a mild enhancement was observed in the numerical results in agreement with that observed in [47]. Crucial to the implementation of SRHND in this model is the assumption that the renormalizable 23 operator is forbidden by unspecified string selection rules, and the leading 23 operator contains $(H \bar{H})$ and involves "Clebsch zeros", which give a zero contribution to the charged lepton and quark Yukawa matrices, but a non-zero contribution to the neutrino Yukawa matrix, thereby allowing small $V_{c b}$ but large 23 mixing in the lepton sector.

The analysis in this paper is essentially "bottom-up". A particular choice of $U(1)_{X}$ family symmetry charges was used to give the horizontal mass splittings, and the vertical mass splittings were achieved by particular choices of operators corresponding to different Clebsch factors in the leading contributions to each entry of the Yukawa matrix. It would be very nice to understand these choices from the point of view of a "top-down" string construction, such as the Type I string construction which has recently led to the Pati-Salam gauge group with three chiral families [20. We believe that only by a combination of top-down and bottom-up approaches (such as that presented here) will a completely successful string theory of fermion masses and mixing angles emerge. We have shown that the recent discovery of neutrino mass by Super-Kamiokande provides precious information about the flavour structure of such a future string theory.

\section{ACKNOWLEDGMENTS}

The work of M.O. was supported by JNICT under contract grant : PRAXIS XXI/BD/ $5536 / 95$. 


\section{APPENDIX A}

TABLE XI

\begin{tabular}{ccrrrr}
\hline \hline CLASS & $\mathcal{O}$ & $x_{u}$ & $x_{d}$ & $x_{e}$ & $x_{\nu}$ \\
\hline I & $\mathcal{O}^{N}$ & 2.0000 & 0.0000 & 0.0000 & 0.0000 \\
I & $\mathcal{O}^{E}$ & 0.0000 & 2.0000 & 0.0000 & 0.0000 \\
I & $\mathcal{O}^{i}$ & 0.0000 & 0.0000 & 2.0000 & 0.0000 \\
I & $\mathcal{O}^{I}$ & 0.0000 & 0.0000 & 0.0000 & 2.0000 \\
II & $\mathcal{O}^{M}$ & 0.0000 & 1.4142 & 1.4142 & 0.0000 \\
II & $\mathcal{O}^{G}$ & 0.0000 & 0.8944 & 1.7889 & 0.0000 \\
II & $\mathcal{O}^{R}$ & 0.0000 & 1.6000 & 1.2000 & 0.0000 \\
II & $\mathcal{O}^{W}$ & 0.0000 & 0.6325 & -1.8974 & 0.0000 \\
III & $\mathcal{O}^{V}$ & 1.4142 & 0.0000 & 0.0000 & 1.4142 \\
III & $\mathcal{O}^{O}$ & 0.8944 & 0.0000 & 0.0000 & 1.7889 \\
III & $\mathcal{O}^{K}$ & 1.6000 & 0.0000 & 0.0000 & 1.2000 \\
III & $\mathcal{O}^{Z}$ & 0.6325 & 0.0000 & 0.0000 & -1.8974 \\
IV & $\mathcal{O}^{J}$ & 0.0000 & 0.0000 & 1.7889 & 0.8944 \\
IV & $\mathcal{O}^{g}$ & 0.0000 & 0.0000 & 1.4142 & 1.4142 \\
IV & $\mathcal{O}^{h}$ & 0.0000 & 0.0000 & -1.4142 & 1.4142 \\
IV & $\mathcal{O}^{j}$ & 0.0000 & 0.0000 & 0.8944 & 1.7889 \\
V & $\mathcal{O}^{F}$ & 1.4142 & -1.4142 & 0.0000 & 0.0000 \\
V & $\mathcal{O}^{a}$ & 1.4142 & 1.4142 & 0.0000 & 0.0000 \\
V & $\mathcal{O}^{b}$ & 1.7889 & 0.8944 & 0.0000 & 0.0000 \\
V & $\mathcal{O}^{c}$ & 0.8944 & 1.7889 & 0.0000 & 0.0000 \\
VI & $\mathcal{O}^{H}$ & 0.8000 & 0.4000 & 0.8000 & 1.6000 \\
VI & $\mathcal{O}^{f}$ & 0.4000 & 0.8000 & 1.6000 & 0.8000 \\
VI & $\mathcal{O}^{S}$ & 0.7155 & 1.4311 & 1.0733 & 0.5367 \\
VI & $\mathcal{O}^{L}$ & 1.4311 & 0.7155 & 0.5367 & 1.0733 \\
VI & $\mathcal{O}^{T}$ & 0.5657 & 0.2828 & 0.2828 & 0.5657 \\
VI & $\mathcal{O}^{U}$ & 0.2828 & 0.5657 & 0.5657 & 0.2828 \\
VI & $\mathcal{O}^{X}$ & 0.5657 & 0.2828 & -0.8485 & -1.6971 \\
VI & $\mathcal{O}^{Y}$ & 0.2828 & 0.5657 & -1.6971 & -0.8485 \\
VI & $\mathcal{O}^{D}$ & 0.4472 & -0.4472 & 1.3416 & -1.3416 \\
VI & $\mathcal{O}^{e}$ & 0.6325 & -0.6325 & -1.2649 & 1.2649 \\
VI & $\mathcal{O}^{B}$ & 1.0000 & -1.0000 & -1.0000 & 1.0000 \\
VI & $\mathcal{O}^{Q}$ & 1.1314 & -1.1314 & -0.8485 & 0.8485 \\
VI & $\mathcal{O}^{P}$ & 1.1314 & 1.1314 & 0.8485 & 0.8485 \\
VI & $\mathcal{O}^{A}$ & 1.0000 & 1.0000 & 1.0000 & 1.0000 \\
VI & $\mathcal{O}^{d}$ & 0.6325 & 0.6325 & 1.2649 & 1.2649 \\
VI & $\mathcal{O}^{C}$ & 0.4472 & 0.4472 & -1.3416 & -1.3416 \\
\hline \hline & & & & & \\
\hline
\end{tabular}

TABLE XI. List of Clebsch factors resulting from all possible $n=1$ operators, as given by Eq. (50), in the Pati-Salam model. 


\section{APPENDIX B}

In this appendix we briefly review some technical issues related to the presence of the right-handed neutrinos. Firstly we show how the decoupling of the neutrinos affects the one-loop RGEs for the Yukawa couplings in the MSSM+ $\nu^{c}$ model :

$$
\begin{aligned}
\frac{1}{16 \pi^{2}} \frac{d \lambda_{u}}{d t} & =\left[3 \operatorname{Tr} U+\operatorname{Tr} N+3 U+D-G^{u}\right] \lambda_{u} \\
\frac{1}{16 \pi^{2}} \frac{d \lambda_{d}}{d t} & =\left[3 \operatorname{Tr} D+\operatorname{Tr} E+3 D+U-G^{d}\right] \lambda_{d} \\
\frac{1}{16 \pi^{2}} \frac{d \lambda_{e}}{d t} & =\left[3 \operatorname{Tr} D+\operatorname{Tr} E+3 E+N-G^{e}\right] \lambda_{e} \\
\frac{1}{16 \pi^{2}} \frac{d \lambda_{\nu}}{d t} & =\left[3 \operatorname{Tr} U+\operatorname{Tr} N+3 N+E-G^{\nu}\right] \lambda_{\nu}
\end{aligned}
$$

where $t=\ln (Q)$,

$$
\begin{array}{ll}
U=\lambda_{u} \lambda_{u}^{\dagger} & G^{u}=\frac{26}{30} g_{1}^{2}+3 g_{2}^{2}+\frac{16}{3} g_{3}^{2} \\
D=\lambda_{d} \lambda_{d}^{\dagger} & G^{d}=\frac{14}{30} g_{1}^{2}+3 g_{2}^{2}+\frac{16}{3} g_{3}^{2} \\
E=\lambda_{e} \lambda_{e}^{\dagger} & G^{e}=\frac{18}{10} g_{1}^{2}+3 g_{2}^{2} \\
N=\lambda_{\nu} \lambda_{\nu}^{\dagger} & G^{\nu}=\frac{6}{10} g_{1}^{2}+3 g_{2}^{2}
\end{array}
$$

and $\operatorname{Tr} U=U_{11}+U_{22}+U_{33}$ etc.. The general idea behind the process of decoupling the right-handed neutrinos (in the "step" approximation) is that a Feynman diagram that includes a specific flavour of a right-handed neutrino $\nu_{A}^{c}$, with mass $M_{\nu_{A}}$, in an internal line only makes a contribution to the RGEs in Eqs. (86)-(89) for energies $Q$ bigger than $M_{\nu_{A}}$. Thus, the procedure depends on properly adapting the $N$ parameter in Eq. (90). We shall now make this statement more precise. Let us assume that the neutrino Majorana matrix $M_{R R}$ is diagonalized by the following transformation :

$$
S^{\nu^{c} \dagger} M_{R R} S^{\nu^{c}}=M_{R R}^{\prime}=\operatorname{diag}\left(M_{\nu_{1}}, M_{\nu_{2}}, M_{\nu_{3}}\right)
$$

Then, the decoupling of the right-handed neutrinos in Eqs. (86)-(89) can be accounted by replacing $N$ in Eq. (90) by $N_{\theta}$ given by :

$$
N=\lambda_{\nu} \lambda_{\nu}^{\dagger}=\lambda_{\nu} S^{\nu^{c}} S^{\nu^{c} \dagger} \lambda_{\nu}^{\dagger} \rightarrow \lambda_{\nu} S^{\nu^{c}} \Theta S^{\nu^{c} \dagger} \lambda_{\nu}^{\dagger}=N_{\theta}
$$

where $\Theta(Q)$ is a energy dependent diagonal matrix defined by :

$$
\Theta(Q)=\operatorname{diag}\left(\theta\left(Q-M_{\nu_{1}}\right), \theta\left(Q-M_{\nu_{2}}\right), \theta\left(Q-M_{\nu_{3}}\right)\right)
$$


with $\theta(x)=0$ for $x<0$ and $\theta(x)=1$ for $x>0$.

The second issue that we would like to address concerns the effect of a large $\left(\lambda_{\nu}\right)_{23}$ coupling on third family Yukawa unification, and as a consequence, for example, on the prediction for the top mass. We claim that the effect is small. To see why let us assume that the only large Yukawa couplings in Eqs. (86)-(89) are $\lambda_{t}=\left(\lambda_{u}\right)_{33}$, $\lambda_{b}=\left(\lambda_{d}\right)_{33}, \lambda_{\tau}=\left(\lambda_{e}\right)_{33}$ and $\lambda_{\nu_{\tau}}=\left(\lambda_{\nu}\right)_{33}, \lambda_{23}=\left(\lambda_{\nu}\right)_{23}$. In this limit, the RGEs simplify to :

$$
\begin{aligned}
\frac{1}{16 \pi^{2}} \frac{d \lambda_{t}}{d t} & =\lambda_{t}\left(6 \lambda_{t}^{2}+\lambda_{b}^{2}+\lambda_{\nu_{\tau}}^{2}+\lambda_{23}^{2}-G^{u}\right) \\
\frac{1}{16 \pi^{2}} \frac{d \lambda_{b}}{d t} & =\lambda_{b}\left(6 \lambda_{b}^{2}+\lambda_{t}^{2}+\lambda_{\tau}^{2}-G^{d}\right) \\
\frac{1}{16 \pi^{2}} \frac{d \lambda_{\tau}}{d t} & =\lambda_{\tau}\left(4 \lambda_{\tau}^{2}+3 \lambda_{b}^{2}+\lambda_{\nu_{\tau}}^{2}-G^{e}\right) \\
\frac{1}{16 \pi^{2}} \frac{d \lambda_{\nu_{\tau}}}{d t} & =\lambda_{\nu_{\tau}}\left(4 \lambda_{\nu_{\tau}}^{2}+4 \lambda_{23}^{2}+3 \lambda_{t}^{2}+\lambda_{\tau}^{2}-G^{\nu}\right)
\end{aligned}
$$

From Eqs. (95),(96) we see that the presence of the $\lambda_{23}$ coupling does not affect the RGEs of $\lambda_{b, \tau}$. Moreover the effect of $\lambda_{23}$ on the RGE of $\lambda_{t}$ is small $(1 / 8 \sim 12 \%$.) The only RGE that is significantly affected by $\lambda_{23}$ is the RGE of $\lambda_{\nu_{\tau}}$. However, since the correct prediction for the heaviest left-handed neutrino $m_{\nu_{3}} \sim 0.05 \mathrm{eV}$ requires that $M_{\nu_{3}}>10^{13} \mathrm{GeV}$, the $\lambda_{\nu_{\tau}}^{2}$ and $\lambda_{23}^{2}$ terms in Eqs. (94),(97) are only present in a rather short energy range, i.e. between $10^{13} \mathrm{GeV}<Q<M_{X} \sim 10^{16} \mathrm{GeV}$. As a consequence the presence/absence of the neutrino Yukawa couplings, as far as third family Yukawa unification is concerned, is not important.

Finally we find interesting to comment on the radiative corrections to the neutrino atmospheric mixing angle $\theta_{23}$ between the GUT and the $M_{Z}$ scale. It is well known [9] that the running of $\sin ^{2}\left(2 \theta_{23}\right)$ can be understood from the following evolution

\footnotetext{
${ }^{24}$ Numerically we found that when $\left(\lambda_{\nu}\right)_{23}$ is allowed to take values comparable with $\left(\lambda_{\nu}\right)_{33}$ the prediction for the top mass roughly decreased by $1 \mathrm{GeV}$, the value of $\tan \beta$ decrease by 0.5 and the value of the unified third family Yukawa coupling at the unification scale decreased by 0.015 .
} 
equation :

$$
\frac{1}{16 \pi^{2}} \frac{1}{\sin ^{2}\left(2 \theta_{23}\right)} \frac{d \sin ^{2}\left(2 \theta_{23}\right)}{d t}=-2\left(\lambda_{\tau}^{2}-\lambda_{\mu}^{2}\right) \frac{\left(m_{L L}\right)_{33}^{2}-\left(m_{L L}\right)_{22}^{2}}{\left[\left(m_{L L}\right)_{33}-\left(m_{L L}\right)_{22}\right]^{2}+4\left(m_{L L}\right)_{23}^{2}}
$$

which displays a resonance peak at $\left(m_{L L}\right)_{33} \sim\left(m_{L L}\right)_{22}$ when $\left(m_{L L}\right)_{23}$ is small. Generally, it is possible that $\left(m_{L L}\right)_{33}$ starts at $Q=M_{X}$ bigger than $\left(m_{L L}\right)_{22}$ but, due to the third family Yukawa radiative effects, to be driven to smaller values faster than $\left(m_{L L}\right)_{22}$. As a result, even if the initial values of $\left(m_{L L}\right)_{33}$ and $\left(m_{L L}\right)_{22}$ at $M_{X}$ are different, they may at some point become comparable. If this is the case then a large $\theta_{23}$ angle can be generated radiatively from a small tree level $\theta_{23}$ at $M_{X}$. This mechanism, of amplifying $\theta_{23}$ radiatively, as been studied for example in Refs. [8, 9]. However, in these works, and as can be seen from Eq. (98), the amplification is only efficient if at least the $\lambda_{\tau}$ Yukawa coupling is large (about 2 or 3.) In our model, since we demanded top-bottom-tau Yukawa unification, the value of the third family Yukawa coupling is rather small $(\sim 0.7)$, thus the $\sin ^{2}\left(2 \theta_{23}\right)$ is stable under radiative corrections. 


\section{APPENDIX C}

In this appendix we show that is easy to convert the results of the LMA MSW solution found in the main body of this paper into results for the LOW solution which is also characterized by maximal $\nu_{e} \rightarrow \nu_{\mu}$ oscillations but smaller $\Delta m_{12}^{2}$ [48, 49]:

$$
\text { LOW : } \quad \sin ^{2}\left(2 \theta_{12}\right) \sim 1 \quad \Delta m_{12}^{2} \sim 10^{-7} \mathrm{eV}^{2}
$$

The reason why we can adapt the LMA results is because, as we showed in section III, in the SRHND approach, the $\theta_{12}$ and $\theta_{23}$ neutrino mixing angles come "solely" from the neutrino Yukawa matrix. On the other hand, the neutrino mass spectrum depends on the hierarchies of $\lambda_{\nu}$ and $M_{R R}$. Thus, as long as we keep within the SRHND scenario, we can change $M_{R R}$ to fit the LOW $\Delta m_{12}^{2}$ solution without that implying a significant change in $\theta_{12}$ and $\theta_{23}$.

Let as consider a LOW model with the same $U(1)_{X}$ flavour charges and the same operator matrix for $\lambda_{f}$ as in the LMA model, given by Table I and Eq. (82), but with a $M_{R R}$ matrix with the following structure :

$$
\frac{M_{R R}\left(M_{X}\right)}{M_{R R}\left(M_{X}\right)_{33}}=\left(\begin{array}{lll}
A_{11} \epsilon^{8} & A_{12} \epsilon^{6} & A_{13} \epsilon^{4} \\
A_{21} \epsilon^{6} & B_{22} \epsilon^{4} & A_{23} \epsilon^{2} \\
A_{31} \epsilon^{4} & A_{32} \epsilon^{2} & A_{33}
\end{array}\right)
$$

Comparing Eq. (100) with Eq. (83) we see that these two equations differ only by their "vertical" $\delta$-component (we assumed that Eq. (100) has $M_{R R}^{\delta} \sim 1$ ) and by the numerical factor $B_{22}=1.821 \neq 1.072=A_{22}$. We note that the removal the $\delta$-factor in the 22 entry of $M_{R R}$ and the increase of the $B_{22}>A_{22}$ coefficient act to decrease $\Delta m_{12}^{2}$.

The Majorana matrix $M_{R R}\left(M_{Z}\right)$ and the neutrino Yukawa matrix $\lambda_{\nu}\left(M_{Z}\right)$ in the LOW model resulting from $M_{R R}\left(M_{X}\right)$ in Eq. (100) and the Yukawa matrices $\lambda_{f}\left(M_{X}\right)$ given by Table IV (recall that we take $\lambda_{f}^{L O W}\left(M_{X}\right)=\lambda_{f}^{L M A}\left(M_{X}\right)$ ) are shown in Table XII. In Table XIII we present the predicted values for the left-handed neutrino 
matrix and the MNS matrix in the LOW model. The results for the neutrino masses in the LOW model are given in Table XIV. Finally in Table XV we show the values of the neutrino mixing angles.

TABLE XII

\begin{aligned} & \hline \hline$\lambda_{\nu}\left(M_{Z}\right)=\left(\begin{array}{ccc}6.120 \times 10^{-6} & 2.271 \times 10^{-3} & 6.368 \times 10^{-2} \\ 2.710 \times 10^{-5} & 2.736 \times 10^{-3} & 0.396 \\ 2.925 \times 10^{-5} & -2.429 \times 10^{-3} & 0.565\end{array}\right) \\ & M_{R R}\left(M_{Z}\right)=\left(\begin{array}{ccc}2.848 \times 10^{9} & 4.036 \times 10^{10} & 7.218 \times 10^{11} \\ 4.036 \times 10^{10} & 2.071 \times 10^{12} & 1.287 \times 10^{13} \\ 7.218 \times 10^{11} & 1.287 \times 10^{13} & 4.551 \times 10^{14}\end{array}\right) \\ &$\hline\end{aligned}

TABLE XII. Numerical values for the entries of the neutrino Yukawa $\left(\lambda_{\nu}\right)$ and Majorana $\left(M_{R R}\right)$ matrices at the $Q=M_{Z}$ scale in the LOW model $\left(M_{R R}\right.$ is given in $\mathrm{GeV}$ mass units.)

\section{TABLE XIII}

\begin{tabular}{|c|c|c|c|c|}
\hline$\frac{m_{L L}\left(M_{Z}\right)}{m_{L L}\left(M_{Z}\right)_{33}}$ & $=$ & $\left(\begin{array}{l}1.334 \times 10^{-2} \\
7.342 \times 10^{-2} \\
9.957 \times 10^{-2}\end{array}\right.$ & $\begin{array}{l}7.342 \times 10^{-2} \\
4.694 \times 10^{-1} \\
6.776 \times 10^{-1}\end{array}$ & $\left.\begin{array}{c}9.957 \times 10^{-2} \\
6.776 \times 10^{-1} \\
1\end{array}\right)$ \\
\hline$V^{M N S}\left(M_{Z}\right)$ & $=$ & $\begin{array}{rr}0.8291 & 0 . \\
-0.3955 & 0 . \\
0.3952 & -0 .\end{array}$ & $\begin{array}{rr}5559 & -0.0597 \\
6586 & 0.6402 \\
.5072 & 0.7659\end{array}$ & \\
\hline
\end{tabular}

TABLE XIII. Predicted values for the left-handed neutrino mass matrix $m_{L L}\left(M_{Z}\right)$ in units of $m_{L L}\left(M_{Z}\right)_{33}=3.567 \times 10^{-3} \mathrm{eV}$ and for the MNS neutrino mixing matrix $V^{M N S}\left(M_{Z}\right)$ in the LOW model.

TABLE XIV

\begin{tabular}{c}
$\overline{m_{\nu_{1}}=7.29 \times 10^{-9} \mathrm{eV} \quad m_{\nu_{2}}=3.54 \times 10^{-4} \mathrm{eV} \quad m_{\nu_{3}}=5.25 \times 10^{-2} \mathrm{eV}}$ \\
$\Delta m_{12}^{2}=1.25 \times 10^{-7} \mathrm{eV}^{2} \quad \Delta m_{23}^{2}=2.76 \times 10^{-3} \mathrm{eV}^{2}$ \\
\hline
\end{tabular}

TABLE XIV. Predicted values for the left-handed neutrino masses $m_{\nu_{1,2,3}}$ and squared mass splittings $\Delta m_{12}^{2}=\left|m_{\nu_{2}}^{2}-m_{\nu_{1}}^{2}\right|, \Delta m_{23}^{2}=\left|m_{\nu_{3}}^{2}-m_{\nu_{2}}^{2}\right|$ in the LOW model. 
TABLE XV

\begin{tabular}{|c|}
\hline $\begin{array}{c}Q=M_{X} \sim 3 \times 10^{16} \mathrm{GeV} \\
\sin \theta_{12}=0.543 \quad \quad \sin \theta_{23}=0.607 \quad \sin \theta_{13}=-0.056 \\
\sin ^{2}\left(2 \theta_{12}\right)=0.832 \quad \sin ^{2}\left(2 \theta_{23}\right)=0.931 \quad \sin ^{2}\left(2 \theta_{13}\right)=0.013\end{array}$ \\
\hline 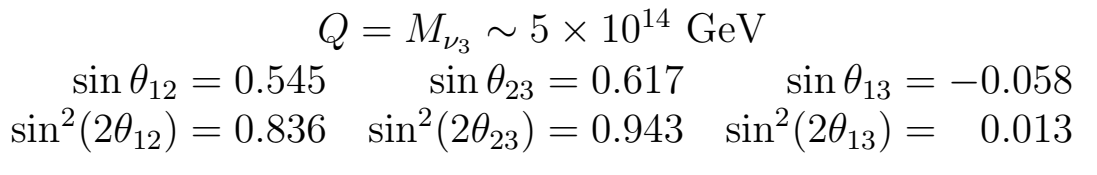 \\
\hline 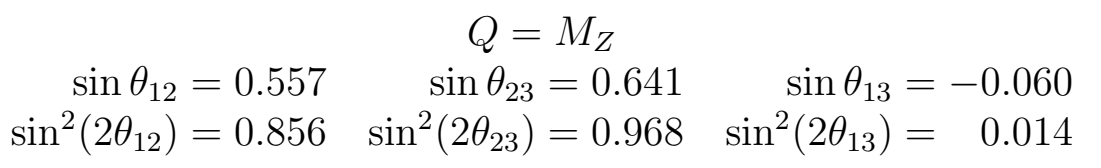 \\
\hline
\end{tabular}

TABLE XV. Running of the neutrino mixing angles at the unification $Q=M_{X}$, the right-handed tau neutrino mass $Q=M_{\nu_{3}}$ and $Z$ boson mass $Q=M_{Z}$ energy scales in the LOW model. 


\section{APPENDIX D}

In this appendix we briefly present a model that explores the possibility of a SMA MSW solution to the solar neutrino anomaly. Although the SMA region is disfavoured by the latest results from the Super-Kamiokande experiment, the SMA solution is not completely ruled out. 20 The SMA solution data indicates 48:

$$
\text { SMA : } \quad \sin ^{2}\left(2 \theta_{12}\right) \sim 1.6 \times 10^{-3} \quad \Delta m_{12}^{2} \sim 5 \times 10^{-6} \mathrm{eV}^{2}
$$

In analogy with the LMA model we start by recalling that the Yukawa and the neutrino Majorana matrices in the SMA model can be decomposed into a "vertical" $\delta$-component and a "horizontal" $\epsilon$-component given by :

$$
\left(\lambda_{f}\right)_{A B} \sim\left(\lambda^{\delta}\right)_{A B}\left(\lambda^{\epsilon}\right)_{A B} \quad\left(M_{R R}\right)_{A B} \sim\left(M_{R R}^{\delta}\right)_{A B}\left(M_{R R}^{\epsilon}\right)_{A B}
$$

\begin{tabular}{|c|c|c|c|c|c|c|c|c|c|}
\hline & $X_{F_{1}}$ & $X_{F_{2}}$ & $X_{F_{3}}$ & $X_{F_{1}^{c}}$ & $X_{F_{2}^{c}}$ & $X_{F_{3}^{c}}$ & $X_{h}$ & $X_{H}$ & $X_{\bar{H}}$ \\
\hline$U(1)_{\bar{X}}$ & 2 & 0 & 0 & 3 & 2 & 0 & 0 & 0 & 0 \\
\hline
\end{tabular}

\section{TABLE XVI}

TABLE XVI. List of the $U(1)_{\bar{X}}$ charges that determine the family structure of the Yukawa and neutrino Majorana matrices in the SMA model.

The $U(1)_{\bar{X}}$ charges of the SMA given in Table XVI fix the "horizontal" structure of $\lambda^{\epsilon}$ and $M_{R R}^{\epsilon}$ in the SMA model to be :

$$
\lambda^{\epsilon} \sim\left(\begin{array}{ccc}
\epsilon^{5} & \epsilon^{4} & \epsilon^{2} \\
\epsilon^{3} & \epsilon^{2} & 1 \\
\epsilon^{3} & \epsilon^{2} & 1
\end{array}\right) \quad M_{R R}^{\epsilon} \sim\left(\begin{array}{ccc}
\epsilon^{6} & \epsilon^{5} & \epsilon^{3} \\
\epsilon^{5} & \epsilon^{4} & \epsilon^{2} \\
\epsilon^{3} & \epsilon^{2} & 1
\end{array}\right)
$$

On the other hand, the "vertical" structure of $\lambda^{\delta}$ and $M_{R R}^{\delta}$ is given by the following operator matrices :

$$
\lambda^{\delta} \sim\left(\begin{array}{ccc}
\mathcal{O}^{G}+\mathcal{O}^{\prime \prime K} & \mathcal{O}^{R}+\mathcal{O}^{\prime O} & \mathcal{O}^{H}+\mathcal{O}^{\prime a} \\
\mathcal{O}^{G}+\mathcal{O}^{\prime \prime V} & \mathcal{O}^{W}+\mathcal{O}^{\prime H} & \mathcal{O}^{I}+\mathcal{O}^{\prime W} \\
\mathcal{O}^{M}+\mathcal{O}^{\prime \prime V} & \mathcal{O}^{g}+\mathcal{O}^{\prime T} & 1
\end{array}\right) \quad M_{R R}^{\delta} \sim \mathbf{1}
$$

\footnotetext{
${ }^{25}$ Statistically, the SMA solution can still describe the neutrino data with a probability of $34 \%$.
} 48 
As a result of Eqs. (103), (104) the Yukawa and the neutrino Majorana matrices in Eq. (102) can be written as :

$$
\begin{aligned}
\lambda_{f}\left(M_{X}\right)= & \left(\begin{array}{ccc}
x_{f}^{G} c_{11} \delta \epsilon^{5} & x_{f}^{R} c_{12} \delta \epsilon^{4} & x_{f}^{H} c_{13} \delta \epsilon^{2} \\
x_{f}^{G} c_{21} \delta \epsilon^{3} & x_{f}^{W} c_{22} \delta \epsilon^{2} & x_{f}^{I} c_{23} \delta \\
x_{f}^{M} c_{31} \delta \epsilon^{3} & x_{f}^{g} c_{32} \delta \epsilon^{2} & c_{33}
\end{array}\right)+ \\
& \left(\begin{array}{ccc}
0 & x_{f}^{O} c_{12}^{\prime} \delta^{2} \epsilon^{4} & x_{f}^{a} c_{13}^{\prime} \delta^{2} \epsilon^{2} \\
0 & x_{f}^{H} c_{22}^{\prime} \delta^{2} \epsilon^{2} & x_{f}^{W} c_{23}^{\prime} \delta^{2} \\
0 & x_{f}^{T} c_{32}^{\prime} \delta^{2} \epsilon^{2} & 0
\end{array}\right)+ \\
& \left(\begin{array}{ccc}
x_{f}^{K} c_{11}^{\prime \prime} \delta^{3} \epsilon^{5} & 0 & 0 \\
x_{f}^{V} c_{21}^{\prime \prime} \delta^{3} \epsilon^{3} & 0 & 0 \\
x_{f}^{V} c_{31}^{\prime \prime} \delta^{3} \epsilon^{3} & 0 & 0
\end{array}\right) \\
\frac{M_{R R}\left(M_{X}\right)}{M_{R R}\left(M_{X}\right)_{33}}= & \left(\begin{array}{lll}
C_{11} \epsilon^{6} & C_{12} \epsilon^{5} & C_{13} \epsilon^{3} \\
C_{21} \epsilon^{5} & C_{22} \epsilon^{4} & C_{23} \epsilon^{2} \\
C_{31} \epsilon^{3} & C_{32} \epsilon^{2} & C_{33}
\end{array}\right)
\end{aligned}
$$

In the rest of this appendix we apply the same systematic approach used in the main part of the paper for the LMA solution to the SMA model. The approximate structure of the effective matrices resulting from Eqs. (105),(106) is given in Table XVII. In Table XVIII we give the values of the $c, C$ parameters appearing in Eqs. (105),(106). In Table XIX we present the exact numerical values of the Yukawa and Majorana matrices at the unification scale and in Table XX the values of the same matrices at the $M_{Z}$ scale. In Table XXI we present the predicted values for the left-handed neutrino mass and for the MNS mixing matrices. The predictions for masses of the physical neutrinos in the SMA model is listed in Table XXII and in Table XXIII we give the predictions for the neutrino mixing angles at several energy scales. Finally, in Table XXIV we show the predictions for the masses of the down and strange quarks in the SMA model. 


\section{TABLE XVII}

\begin{tabular}{|c|c|c|c|c|c|c|c|c|}
\hline \multirow{3}{*}{$\lambda_{u}\left(M_{X}\right)$} & \multirow{3}{*}{$\sim$} & $\left(\delta^{3} \epsilon^{5}\right.$ & $\delta^{2} \epsilon^{4}$ & $\left.\delta \epsilon^{2}\right)$ & \multirow{3}{*}{$\sim$} & $\left(\lambda^{8}\right.$ & $\lambda^{6}$ & $\left.\lambda^{3}\right)$ \\
\hline & & $\delta^{3} \epsilon^{3}$ & $\delta^{2} \epsilon^{2}$ & 0 & & $\lambda^{6}$ & $\lambda^{4}$ & 0 \\
\hline & & $\delta^{3} \epsilon^{3}$ & $\delta^{2} \epsilon^{2}$ & 1 & & $\lambda^{6}$ & $\lambda^{4}$ & 1 \\
\hline \multirow{3}{*}{$\lambda_{d}\left(M_{X}\right)$} & \multirow{3}{*}{$\sim$} & $\delta \epsilon^{5}$ & $\delta \epsilon^{4}$ & $\delta \epsilon^{2}$ & \multirow{3}{*}{$\sim$} & $\left(\lambda^{6}\right.$ & $\lambda^{5}$ & $\left.\lambda^{3}\right)$ \\
\hline & & $\delta \epsilon^{3}$ & $\delta \epsilon^{2}$ & $\delta^{2}$ & & $\lambda^{4}$ & $\lambda^{3}$ & $\lambda^{2}$ \\
\hline & & $\delta \epsilon^{3}$ & $\delta^{2} \epsilon^{2}$ & 1 & & $\lambda^{4}$ & $\lambda^{4}$ & $1)$ \\
\hline \multirow{3}{*}{$\lambda_{e}\left(M_{X}\right)$} & \multirow{3}{*}{$\sim$} & $\delta \epsilon^{5}$ & $\delta \epsilon^{4}$ & $\delta \epsilon^{2}$ & \multirow{3}{*}{$\sim$} & $\left(\lambda^{6}\right.$ & $\lambda^{5}$ & $\lambda^{3}$ \\
\hline & & $\delta \epsilon^{3}$ & $\delta \epsilon^{2}$ & $\delta^{2}$ & & $\lambda^{4}$ & $\lambda^{3}$ & $\lambda^{2}$ \\
\hline & & $\delta \epsilon^{3}$ & $\delta \epsilon^{2}$ & 1 & & $\lambda^{4}$ & $\lambda^{3}$ & $1)$ \\
\hline \multirow{3}{*}{$\lambda_{\nu}\left(M_{X}\right)$} & \multirow{3}{*}{$\sim$} & $\left(\delta^{3} \epsilon^{5}\right.$ & $\delta^{2} \epsilon^{4}$ & $\delta \epsilon^{2}$ & \multirow{3}{*}{$\sim$} & $\left(\lambda^{8}\right.$ & $\lambda^{6}$ & $\lambda^{3}$ \\
\hline & & $\delta^{3} \epsilon^{3}$ & $\delta^{2} \epsilon^{2}$ & $\delta$ & & $\lambda^{6}$ & $\lambda^{4}$ & $\lambda$ \\
\hline & & $\delta^{3} \epsilon^{3}$ & $\delta \epsilon^{2}$ & 1 & & $\lambda^{6}$ & $\lambda^{3}$ & 1 \\
\hline \multirow{3}{*}{$M_{R R}\left(M_{X}\right)$} & \multirow{3}{*}{$\sim$} & $\left(\epsilon^{6}\right.$ & $\epsilon^{5}$ & $\epsilon^{3}$ & \multirow{3}{*}{$\sim$} & $\left(\lambda^{6}\right.$ & $\lambda^{5}$ & $\left.\lambda^{3}\right)$ \\
\hline & & $\epsilon^{5}$ & $\epsilon^{4}$ & $\epsilon^{2}$ & & $\lambda^{5}$ & $\lambda^{4}$ & $\lambda^{2}$ \\
\hline & & $\epsilon^{3}$ & $\epsilon^{2}$ & 1 & & $\lambda \lambda^{3}$ & $\lambda^{2}$ & $1)$ \\
\hline
\end{tabular}

TABLE XVII. Approximate structure of the Yukawa and neutrino Majorana matrices in the SMA model resulting from Eqs. (105), (106) when the numerical effect of the Clebsch and of the $c, C$ parameters is neglected.

TABLE XVIII

\begin{aligned} \hline \hline & $=\left(\begin{array}{rrr}-1.403 & -1.656 & 1.000 \\ 1.000 & -1.563 & 1.000 \\ 1.000 & 1.000 & 0.682\end{array}\right) \\ c^{\prime} & =\left(\begin{array}{rrr}0.000 & 1.854 & 1.000 \\ 0.000 & -0.807 & 0.702 \\ 0.000 & 1.000 & 0.000\end{array}\right) \\ c^{\prime \prime} & =\left(\begin{array}{rrr}-1.131 & 0.000 & 0.000 \\ 1.000 & 0.000 & 0.000 \\ 1.000 & 0.000 & 0.000 \\ 1.069 & 0.533 & 0.799 \\ 0.533 & 1.054 & 0.753 \\ 0.799 & 0.753 & 1.000\end{array}\right) \\ C= & \end{aligned}$

TABLE XVIII. Numerical values of the order-one $c, C$ parameters that parameterise the Yukawa and neutrino Majorana matrices of Eqs. (105),(106) in the SMA model. 
TABLE XIX

\begin{tabular}{|c|c|c|c|c|}
\hline \multirow{3}{*}{$\lambda_{u}\left(M_{X}\right)$} & \multirow{3}{*}{$=$} & $\left(-9.935 \times 10^{-6}\right.$ & $1.880 \times 10^{-4}$ & $1.183 \times 10^{-2}$ \\
\hline & & $1.603 \times 10^{-4}$ & $-1.511 \times 10^{-3}$ & 0.000 \\
\hline & & $1.603 \times 10^{-4}$ & $1.325 \times 10^{-3}$ & 0.682 \\
\hline \multirow{3}{*}{$\lambda_{d}\left(M_{X}\right)$} & \multirow{3}{*}{$=$} & $\left(-1.423 \times 10^{-4}\right.$ & $-1.365 \times 10^{-3}$ & $\left.7.572 \times 10^{-3}\right)$ \\
\hline & & $2.095 \times 10^{-3}$ & $-1.128 \times 10^{-2}$ & $2.149 \times 10^{-2}$ \\
\hline & & $3.313 \times 10^{-3}$ & $6.626 \times 10^{-4}$ & 0.682 \\
\hline \multirow{3}{*}{$\lambda_{e}\left(M_{X}\right)$} & \multirow{3}{*}{$=$} & $\left(-2.846 \times 10^{-4}\right.$ & $-1.024 \times 10^{-3}$ & $8.518 \times 10^{-3}$ \\
\hline & & $4.190 \times 10^{-3}$ & $3.006 \times 10^{-2}$ & $-6.447 \times 10^{-2}$ \\
\hline & & $3.313 \times 10^{-3}$ & $1.572 \times 10^{-2}$ & 0.683 \\
\hline \multirow{3}{*}{$\lambda_{\nu}\left(M_{X}\right)$} & \multirow{3}{*}{$=$} & $\left(-7.451 \times 10^{-6}\right.$ & $3.760 \times 10^{-4}$ & $1.704 \times 10^{-2}$ \\
\hline & & $1.603 \times 10^{-4}$ & $-3.023 \times 10^{-3}$ & 0.440 \\
\hline & & $1.603 \times 10^{-4}$ & $1.638 \times 10^{-2}$ & 0.682 \\
\hline \multirow{3}{*}{$M_{R R}\left(M_{X}\right)$} & \multirow{3}{*}{$=$} & $1.177 \times 10^{11}$ & $2.664 \times 10^{11}$ & $8.261 \times 10^{12}$ \\
\hline & & $2.664 \times 10^{11}$ & $2.398 \times 10^{12}$ & $3.541 \times 10^{13}$ \\
\hline & & $8.261 \times 10^{12}$ & $3.541 \times 10^{13}$ & $9.708 \times 10^{14}$ \\
\hline
\end{tabular}

TABLE XIX. Numerical values for the entries of the Yukawa $\left(\lambda_{u}, \lambda_{d}, \lambda_{e}, \lambda_{\nu}\right)$ and neutrino Majorana $\left(M_{R R}\right)$ matrices at the unification scale $M_{X}$ in the SMA model $\left(M_{R R}\right.$ is given in $\mathrm{GeV}$ mass units.)

TABLE XX

\begin{tabular}{|c|c|c|c|c|}
\hline \multirow{3}{*}{$\lambda_{u}\left(M_{Z}\right)$} & \multirow{3}{*}{$=$} & $\left(-2.323 \times 10^{-5}\right.$ & $3.837 \times 10^{-4}$ & $1.644 \times 10^{-2}$ \\
\hline & & $3.409 \times 10^{-4}$ & $-3.228 \times 10^{-3}$ & $-3.588 \times 10^{-3}$ \\
\hline & & $2.125 \times 10^{-4}$ & $1.767 \times 10^{-3}$ & 0.907 \\
\hline \multirow{3}{*}{$\lambda_{d}\left(M_{Z}\right)$} & \multirow{3}{*}{$=$} & $\left(-3.155 \times 10^{-4}\right.$ & $-2.734 \times 10^{-3}$ & $8.864 \times 10^{-3}$ \\
\hline & & $4.130 \times 10^{-3}$ & $-2.256 \times 10^{-2}$ & $3.088 \times 10^{-2}$ \\
\hline & & $4.162 \times 10^{-3}$ & $1.053 \times 10^{-3}$ & 0.864 \\
\hline \multirow{3}{*}{$\lambda_{e}\left(M_{Z}\right)$} & \multirow{3}{*}{$=$} & $\left(-2.846 \times 10^{-4}\right.$ & $-1.032 \times 10^{-3}$ & $6.511 \times 10^{-3}$ \\
\hline & & $4.090 \times 10^{-3}$ & $2.923 \times 10^{-2}$ & $-5.322 \times 10^{-2}$ \\
\hline & & $2.619 \times 10^{-3}$ & $1.257 \times 10^{-2}$ & 0.526 \\
\hline \multirow{3}{*}{$\lambda_{\nu}\left(M_{Z}\right)$} & \multirow{3}{*}{$=$} & $\left(-7.251 \times 10^{-6}\right.$ & $3.290 \times 10^{-4}$ & $1.481 \times 10^{-2}$ \\
\hline & & $1.464 \times 10^{-4}$ & $-2.961 \times 10^{-3}$ & 0.399 \\
\hline & & $1.341 \times 10^{-4}$ & $1.387 \times 10^{-2}$ & 0.572 \\
\hline \multirow{3}{*}{$M_{R R}\left(M_{Z}\right)$} & \multirow{3}{*}{$=$} & $1.176 \times 10^{11}$ & $2.629 \times 10^{11}$ & $8.034 \times 10^{12}$ \\
\hline & & $2.629 \times 10^{11}$ & $2.370 \times 10^{12}$ & $3.409 \times 10^{13}$ \\
\hline & & $8.034 \times 10^{12}$ & $3.409 \times 10^{13}$ & $9.197 \times 10^{14}$ \\
\hline
\end{tabular}

TABLE XX. Numerical values for the entries of the Yukawa $\left(\lambda_{u}, \lambda_{d}, \lambda_{e}, \lambda_{\nu}\right)$ and neutrino Majorana $\left(M_{R R}\right)$ matrices at the $Q=M_{Z}$ scale in the SMA model $\left(M_{R R}\right.$ is given in $\mathrm{GeV}$ mass units.) 


\section{TABLE XXI}

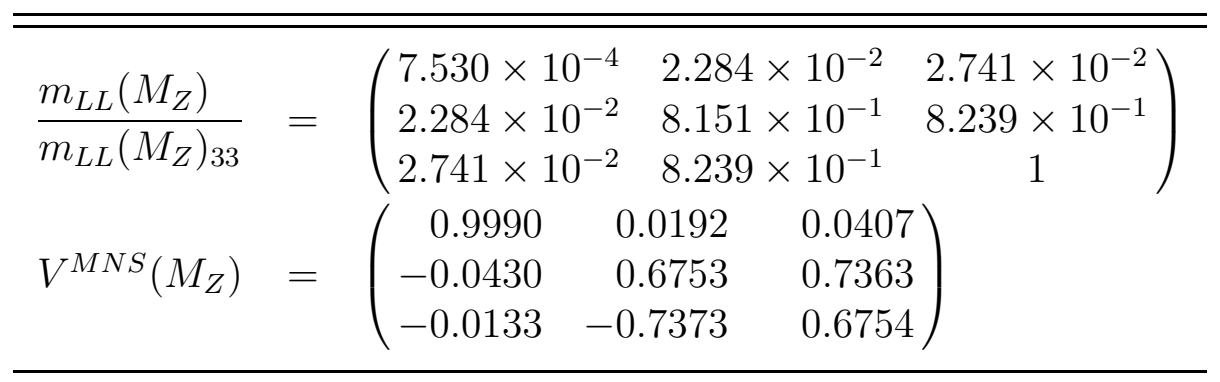

TABLE XXI. Predicted values for the left-handed neutrino mass matrix $m_{L L}\left(M_{Z}\right)$ in units of $m_{L L}\left(M_{Z}\right)_{33}=2.893 \times 10^{-3} \mathrm{eV}$ and for the MNS neutrino mixing matrix $V^{M N S}\left(M_{Z}\right)$ in the SMA model.

\section{TABLE XXII}

$\overline{m_{\nu_{1}}=3.07 \times 10^{-8} \mathrm{eV} \quad m_{\nu_{2}}=2.27 \times 10^{-3} \mathrm{eV} \quad m_{\nu_{3}}=5.03 \times 10^{-2} \mathrm{eV}}$
$\Delta m_{12}^{2}=5.15 \times 10^{-6} \mathrm{eV}^{2} \quad \Delta m_{23}^{2}=2.52 \times 10^{-3} \mathrm{eV}^{2}$

TABLE XXII. Predicted values for the left-handed neutrino masses $m_{\nu_{1,2,3}}$ and squared mass splittings $\Delta m_{12}^{2}=\left|m_{\nu_{2}}^{2}-m_{\nu_{1}}^{2}\right|, \Delta m_{23}^{2}=\left|m_{\nu_{3}}^{2}-m_{\nu_{2}}^{2}\right|$ in the SMA model.

\section{TABLE XXIII}

\begin{tabular}{|c|c|}
\hline $\begin{aligned} \sin \theta_{12} & =2.17 \times 10^{-2} \\
\sin ^{2}\left(2 \theta_{12}\right) & =1.87 \times 10^{-3}\end{aligned}$ & $\begin{aligned}=M_{X} \sim 3 & \times 10^{16} \mathrm{GeV} \\
\sin \theta_{23} & =0.703 \quad \sin \theta_{13}=3.87 \times 10^{-2} \\
\sin ^{2}\left(2 \theta_{23}\right) & =1.000 \quad \sin ^{2}\left(2 \theta_{13}\right)=5.97 \times 10^{-3}\end{aligned}$ \\
\hline $\begin{aligned} \sin \theta_{12} & =2 \\
\sin ^{2}\left(2 \theta_{12}\right) & =1\end{aligned}$ & $\begin{array}{lr}.713 & \sin \theta_{13} \\
.000 & \sin ^{2}\left(2 \theta_{13}\right)\end{array}$ \\
\hline $\begin{array}{r}\sin \theta_{12}= \\
\sin ^{2}\left(2 \theta_{12}\right)=\end{array}$ & $\begin{array}{cc}Q=M_{Z} & \\
\sin \theta_{23}=0.737 & \sin \\
\ln ^{2}\left(2 \theta_{23}\right)=0.993 & \sin ^{2}(2 \theta\end{array}$ \\
\hline
\end{tabular}

TABLE XXIII. Running of the neutrino mixing angles at the unification $Q=M_{X}$, the right-handed tau neutrino mass $Q=M_{\nu_{3}}$ and $Z$ boson mass $Q=M_{Z}$ energy scales in the SMA model. 
TABLE XXIV

$\overline{\overline{m_{d}(1 \mathrm{GeV})=7.6 \mathrm{MeV} \quad m_{s}\left(M_{s}\right)=193 \mathrm{MeV}}}$

TABLE XXIV. Predictions for the running $\overline{\mathrm{MS}}$ masses of the down $\left(m_{d}\right)$ and strange $\left(m_{s}\right)$ quarks at $Q=1 \mathrm{GeV}$ and $Q=M_{s}$ respectively in the SMA model $\left(M_{s}\right.$ indicates the strange pole quark mass.) 


\section{References}

[1] Y. Fukuda et al., Super-Kamiokande Collaboration, Phys. Lett. B433, 9 (1998); ibid. Phys. Lett. B436, 33 (1998); ibid. Phys. Rev. Lett. 81, 1562 (1998).

[2] H. Sobel, talk presented at the XIX International Conference on Neutrino Physics and Astrophysics, Sudbury, Canada, June 16-21, 2000.

[3] L. Wolfenstein, Phys. Rev. D17, 2369 (1978); ibid. Phys. Rev. D20, 2634 (1979); S. P. Mikheyev, A. Y. Smirnov, Yad. Fiz. 42, 1441 (1985) [Sov. J. Nucl. Phys. 42, 913 (1985)]; Nuovo Cimento 9C, 17 (1986).

[4] Y. Suzuki, talk presented at the XIX International Conference on Neutrino Physics and Astrophysics, Sudbury, Canada, June 16-21, 2000.

[5] J. N. Bahcall, P. I. Krastev, A. Y. Smirnov, Phys. Rev. D60 093001 (1999) hep-ph/9905220.

[6] M. Gell-Mann, P. Ramond, R. Slansky, in Sanibel Talk, CALT-68-709, Feb. 1979 and in Supergravity (North Holland, Amsterdam 1979); T. Yanagida in Proc. of the Workshop on Unified Theory and Baryon Number of the Universe, KEK, Japan (1979); R. N. Mohapatra, G. Senjanovic, Phys. Rev. Lett. 44, 912 (1980).

[7] S. F. King, N. N. Singh, hep-ph/0007243.

[8] J. Ellis, G. K. Leontaris, S. Lola, D. V. Nanopoulos, Eur. Phys. J. C9, 389 (1999);

[9] K. S. Babu, C. N. Leung, J. Pantaleone, Phys. Lett. B319, 191 (1993); M. Tanimoto, Phys. Lett. B360, 41 (1995).

[10] S. F. King, Phys. Lett. B439, 350 (1998).

[11] S. F. King, Nucl. Phys. B562, 57 (1999). 
[12] S. F. King, Nucl. Phys. B576, 85 (2000).

[13] G. Altarelli, F. Feruglio, Phys. Lett. B451, 388 (1999).

[14] G. Altarelli, F. Feruglio, I. Masina, Phys. Lett. B472, 382 (2000).

[15] K. S. Babu, J. C. Pati, F. Wilczek, Nucl. Phys. B566, 33 (2000).

[16] C. H. Albright, S. M. Barr, Phys. Rev. Lett. 85, 244 (2000).

[17] Q. Shafi, Z. Tavartkiladze, Phys. Lett. B487, 145 (2000).

[18] J. C. Pati, A. Salam, Phys. Rev. D10, 275 (1974).

[19] I. Antoniadis and G. K. Leontaris, Phys. Lett. B216, 333 (1989); I. Antoniadis and G. K. Leontaris, and J. Rizos, Phys. Lett. B245, 161 (1990).

[20] G. Shiu, S. H. H. Tye, Phys. Rev. D58, 106007 (1998).

[21] S. F. King, Phys. Lett. B325, 129 (1994).

[22] B. C. Allanach, S.F. King, Nucl. Phys. B456, 57 (1995).

[23] B. C. Allanach, S. F. King, Nucl. Phys. B459, 75 (1996).

[24] B. C. Allanach, S. F. King, G. K. Leontaris, S. Lola, Phys. Rev. D56, 2632 (1997).

[25] S. F. King, M. Oliveira, hep-ph/0008183.

[26] H. P. Nilles, Phys. Rep. 110, 1 (1984) ; H. E. Haber and G. L. Kane, Phys. Rep. 117, 75 (1985) ; A. B. Lahanas and D. V. Nanopoulos, Phys. Rep 145, 1 (1987); H. E. Haber, Presented at Theoretical Advanced Study Institute (TASI 92): From Black Holes and Strings to Particles, Boulder, CO, 3-28 Jun 1992. Published in Boulder TASI 92:0589-688, hep-ph/9305248. 
[27] N. Cabibbo, Phys. Rev. Lett. 10, 531 (1963); M. Kobayashi, T. Maskawa, Prog. Theor. Phys. 49, 652 (1973).

[28] Z. Maki, M. Nakagawa, S. Sakata, Prog. Theor. Phys. 28, 870 (1962).

[29] S. F. King and Q. Shafi, Phys. Lett. B422, 135 (1998).

[30] P. Ramond, R. G. Roberts, G. G. Ross, Nucl. Phys. B406, 19 (1993); Y. Grossman, Y. Nir, Nucl. Phys. B448, 30 (1995); M. Leurer, Y. Nir, N. Seiberg, Nucl. Phys. B398, 319 (1993); ibid., Nucl. Phys. B420, 468 (1994).

[31] L. Ibáñez, G. G. Ross, Phys. Lett. B332, 100 (1994).

[32] V. Jain, R. Shrock, Phys. Lett. B352, 83 (1995).

[33] P. Binetruy, P. Ramond, Phys. Lett. B350, 49 (1995); E. Dudas, S. Pokorski, C.A. Savoy, Phys. Lett. B356, 45 (1995); P. Binétruy, S. Lavignac, P. Ramond, Nucl. Phys. B477, 353 (1996); E. J. Chun, A. Lukas, Phys. Lett. B387, 99 (1996).

[34] H. Dreiner, G. K. Leontaris, S. Lola, G. G. Ross, C. Scheich, Nucl. Phys. B436, 461 (1995); G. K. Leontaris, S. Lola ,C. Scheich, J. D. Vergados, Phys. Rev. D53, 6381 (1996); S. Lola, G. G. Ross, Nucl. Phys. B553, 81 (1999); M. Carena, J. Ellis, S. Lola, C. E. M. Wagner, Eur. Phys. J. C12, 507 (2000).

[35] R. Barbieri, L. J. Hall, D. Smith, A. Strumia, N. Weiner, JHEP 9812, 17 (1998); Y. Grossman, Y. Nir, Y. Shadmi, JHEP 9810, 007 (1998); G. Altarelli, F. Feruglio, Phys. Lett. B439, 112 (1998);

[36] S. M. Barr, I. Dorsner, hep-ph/0003058.

[37] L. Wolfenstein, Phys. Rev. Lett. 51, 1945 (1983).

[38] B. C. Allanach, S. F. King, Nucl. Phys. B507, 91 (1997). 
[39] M. Green, J. Schwarz, Phys. Lett. 149, 117 (1984).

[40] L. E. Ibáñez, Phys. Lett. B303, 55 (1993).

[41] H. Georgi, C. Jarlskog, Phys. Lett. B86 297 (1979); S. Dimopoulos, L. J. Hall, S. Raby, Phys. Rev. D45, 4192 (1992).

[42] C. D. Froggatt, H. B. Nielsen, Nucl. Phys. B147, 277 (1979).

[43] G. Anderson, S. Raby, S. Dimopoulos, L.J. Hall, G.D. Starkman, Phys. Rev. D49, 3660 (1994).

[44] D. E. Groom, et al. (Particle Data Group), Eur. Phys. Jour. C15, 1 (2000).

[45] H. Arason, D. J. Castaño, B. Kesthelyi, S. Mikaelian, E. J. Piard, P. Ramond and B. D. Wright, Phys. Rev. D46, 3945 (1992).

[46] M. Apollonio et al. (CHOOZ collaboration), Phys. Lett. B466, 415, (1999); ibid. Phys. Lett. B420, 397 (1998).

[47] S .F. King and N. N. Singh, hep-ph/0006229, Nucl. Phys. B (to appear).

[48] M. C. Gonzalez-Garcia and C. Peña-Garay, hep-ph/0009041.

[49] J. Ellis, Talk given at the 19TH International Conference on Neutrino Physics and Astrophysics - Neutrino 2000, Sudbury, Ontario, Canada, 16-21 Jun 2000. hep-ph/0008334 